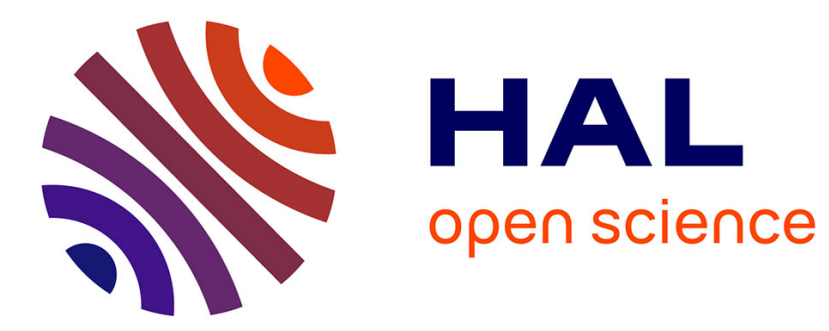

\title{
Résilience économique et disparité territoriale
}

\author{
Magali Talandier, Yatina Calixte
}

\section{To cite this version:}

Magali Talandier, Yatina Calixte. Résilience économique et disparité territoriale: Quelles leçons retenir de la crise de 2008?. Revue d'économie régionale et urbaine, 2021, Juin (3), pp.361-396. 10.3917/reru.213.0361 . hal-03312726

\section{HAL Id: hal-03312726 \\ https://hal.science/hal-03312726}

Submitted on 2 Aug 2021

HAL is a multi-disciplinary open access archive for the deposit and dissemination of scientific research documents, whether they are published or not. The documents may come from teaching and research institutions in France or abroad, or from public or private research centers.
L'archive ouverte pluridisciplinaire HAL, est destinée au dépôt et à la diffusion de documents scientifiques de niveau recherche, publiés ou non, émanant des établissements d'enseignement et de recherche français ou étrangers, des laboratoires publics ou privés. 
See discussions, stats, and author profiles for this publication at: https://www.researchgate.net/publication/348742596

Résilience économique et disparité territoriale : Quelles leçons retenir de la crise de 2008 ?

Preprint · January 2021

CITATIONS

0

2 authors:

2. Magali Talandier

Université Grenoble Alpes

70 PUBLICATIONS 239 CITATIONS

SEE PROFILE

Some of the authors of this publication are also working on these related projects:

Project

Small and medium sized cities issues View project

Project Urban innovation // Rural innovation View project
READS

1,324

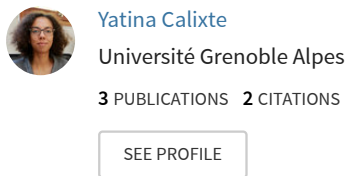


Article accepté et à paraitre dans la RERU (Revue d'Economie Régionale et Urbaine), Vol (2) ou Vol (3), courant 2021

\title{
Résilience économique et disparité territoriale : Quelles leçons retenir de la crise de 2008 ?
}

\section{Economic resilience of French territories:}

What lessons can be learned from the 2008 crisis?

\author{
Magali TALANDIER \\ Université Grenoble Alpes, UMR PACTE \\ magali.talandier@univ-grenoble-alpes.fr \\ Auteur correspondant

\section{Yatina CALIXTE} \\ Université Grenoble Alpes, UMR PACTE \\ yatina.calixte@aurg.asso.fr
}




\title{
Mots-clés
}

Résilience ; développement territorial ; crise économique ; intercommunalités ; métropole.

\section{Keywords}

Resilience ; territorial development ; economic crisis ; intermunicipalities ; metropolis.

\section{Classification JEL : R11}

\section{Résumé}

La crise économique de 2008 a été l'une des plus sévères que l'Europe ait connu jusque-là. L'impact de la crise a été extrêmement variable d'une région à l'autre au niveau européen, questionnant les enjeux de solidarité et de cohésion territoriale. Ainsi, alors qu'une nouvelle crise économique émerge suite à la pandémie de la Covid-19, tous les territoires n'ont pas encore retrouvé leurs niveaux d'emplois de 2007. Dans ce contexte, l'article interroge la capacité de résilience économique des territoires, à l'échelle des intercommunalités de France métropolitaine. Le fait de disposer de dix années d'observation fine des variations de l'emploi privé nous permet de comparer l'intensité des pertes, puis les capacités de rebond, ainsi que d'introduire la temporalité de ces effets à travers la notion de cycle de résilience. Les résultats montrent la diversité des trajectoires économiques et les disparités territoriales en termes de résilience, questionnant dès lors les possibles impacts spatiaux de la crise actuelle.

\begin{abstract}
The economic crisis of 2008 was one of the most severe that Europe has experienced so far. The impact of the crisis has been extremely variable from one region to another at European level, raising the issues of solidarity and territorial cohesion. Thus, while a new crisis is coming linked to the coronavirus pandemic, not all territories have yet recovered their 2007 employment levels. In this context, the article examines the economic resilience of the territories, at the level of the intermunicipalities in France. To have ten years of detailed observation of variations in private employment allows us to compare not only employment dynamics, but also the intensity of losses and then the capacity to rebound, as well as to introduce the temporality of these effects through the notion of the resilience cycle. The results show the diversity of economic trajectories and territorial disparities in terms of resilience. Disparities, in terms of resilience, are more marked between regions than between metropolises and the rest of the territory in the short term. Over the long term, the "metropolis-hinterland" divide within the regions hardest hit by the crisis is increasing. In most of these areas, employment certainly began to rise again after 2009, but more slowly than elsewhere, not enough to make up for the losses. Here, the COVID-19 crisis may not be stronger than elsewhere, but it will hit areas (and households) that were already highly precarious at the start of the pandemic.
\end{abstract}

\section{Points clés}

- Nous examinons l'impact de la crise de 2008 afin de mieux comprendre les inégalités spatiales de résilience économique (effet métropolitain versus effet régional).

- Nous traitons les séries temporelles d'emplois privés en termes d'intensité et, de façon plus innovante sur le cas français, de temporalité de résilience.

- Les disparités de résilience sont plus marquées entre les régions qu'entre les métropoles et le reste du territoire à court terme.

- Sur la longue période, se renforce la fracture «métropole-hinterland » au sein des régions les plus durement touchées par la crise. 


\section{$-1-$ \\ Introduction}

La période de ralentissement économique de 2008 a été l'une des pires crises économiques qu'ait connue l'Europe (Capello et Caragliu, 2016). L'effondrement de la bulle immobilière américaine et le recours excessif aux crédits ont provoqué une récession sans précédent qui s'est répandue à travers toute l'Europe (Christopherson et al., 2015). Les racines de cette crise européenne sont une combinaison entre une perte de compétitivité et un haut niveau d'endettement, spécialement dans les pays périphériques de l'Union Européenne monétaire (Crescendi et al., 2016).

Ainsi, alors même qu'une nouvelle crise économique majeure s'annonce, suite à la pandémie de la COVID-19, tous les pays européens n'ont pas encore récupéré de la crise précédente. Ce constat est encore plus vrai aux échelles infranationales, risquant de provoquer et/ou de renforcer certaines inégalités spatiales. Tirer aujourd'hui les leçons de la crise de 2008 en termes de résilience économique des territoires s'avère encore plus urgent qu'avant la pandémie.

La crise de 2008 s'est diffusée de façon asymétrique dans le temps et à travers les régions de l'UE (Capello et al. 2015 ; Groot et a., 2011). Toutes n'ont pas connu un déclin économique et les impacts territoriaux varient grandement à l'intérieur d'un même pays (Martin, 2010). Tandis que certaines régions ont connu un rapide retour au niveau d'emplois d'avant la crise, le processus de récupération a été beaucoup plus long, voire même inachevé pour d'autres.

Les travaux développés sur le sujet montrent que la crise a renforcé une Europe à plusieurs vitesses (Capello et al., 2015). Les régions des pays du Sud de l'Europe ont été très touchées, tandis que les pays du Nord furent moins impactés. La pandémie actuelle risque de renforcer ces inégalités. Les pays de l'Est connaissent, quant à eux, une croissance moins rapide depuis 2008 et enregistrent une hausse des inégalités inter-régionales. À une échelle infranationale, tous les travaux ont conclu à une meilleure résistance des régions capitales (Pontarollo et Serpieri, 2020) et, plus largement, des régions métropolitaines (Capello et al., 2015). C'est notamment le cas pour l'Allemagne (Berlin, Munich, et Frankfurt), le Danemark (Copenhague), la Suède (Stockholm, Göteborg et Malmö), l'Espagne (Madrid, Barcelona et Séville) ou l'Italie (Rome, Milan et Turin).

Cet avantage métropolitain génère une augmentation des disparités spatiales au sein de chaque pays. Le vrai enjeu tient alors dans le choix des politiques publiques pour résorber ces inégalités. Faut-il soutenir les régions périphériques, a priori plus touchées par la crise, ou au contraire renforcer les métropoles pour assurer la croissance globale des régions (Camagni et Capello, 2015) ? L'OCDE, conforté par le Rapport Barca à la Commission européenne (Barca, 2009), défend depuis longtemps un système basé sur une logique de développement des régions périphériques, en fonction des spécificités des lieux, de la mobilisation locale avec le soutien d'acteurs institutionnels et économiques externes (Coffano et Foray, 2014 ; McCann et OrtegaArgiles, 2014). L'autre logique, redistributive, est soutenue par la Banque mondiale. Elle consiste à souligner l'efficacité des grandes zones métropolitaines et la nécessité de les soutenir au nom du bien-être global (Sapir et al., 2004). Cette deuxième approche, qui n'est pas neutre sur le plan spatial, fait l'objet de vives critiques (Barca et al., 2012).

En France, ce type de débat a eu lieu. Assez étonnamment, il a moins porté sur la question des inégalités, des fractures et des politiques de rééquilibrage à privilégier pour les résorber, que sur le fait de savoir si oui ou non les métropoles étaient réellement plus compétitives que les autres territoires (Bouba-Olga, Grossetti, 2015). 
Si au niveau national, la France apparait dans une situation intermédiaire, entre l'Europe du Sud fortement touchée et l'Europe du Nord, moins impactée, au niveau infranational, les résultats sont plus contrastés. Les premiers travaux ont rapidement conforté les analyses européennes qui montraient la plus grande capacité de résistance des métropoles (FNAU, 2011 ; Davezies, 2012 ; Borzic et Le Jeannic, 2014 ; Baude, 2016). Puis, d'autres études sont venues tempérer ces conclusions en insistant essentiellement sur la diversité des territoires (Bouba-Olga, 2017).

Finalement, 10 ans après la crise de 2008, et à l'amorce d'une nouvelle récession, la question de la plus grande capacité de résilience des métropoles françaises reste posée, et avec elle, celle des disparités spatiales face à la crise. Les métropoles sont-elles réellement plus résilientes que les autres territoires ? Quelles leçons en tirer pour la crise actuelle ${ }^{1}$ ?

Notre première hypothèse repose sur l'idée que la crise de 2008 aurait encore plus accéléré les fractures régionales que celles entre les métropoles et leur périphérie. Autrement dit, l'effet métropolitain serait moins déterminant que l'effet structurel régional. Cependant, notre seconde hypothèse postule que ce type d'impact peut varier dans le temps : les métropoles toujours très fragilisées au moment d'un choc économique, retrouvent ensuite une croissance rapide des emplois ou des revenus.

Notre article repose sur trois sections distinctes. Tout d'abord, nous définirons le concept de résilience économique et ses facteurs (section 2). Puis, nous expliciterons notre méthodologie en termes de choix des variables, d'échelle d'analyse et d'indicateurs (section 3). Enfin, nous présenterons et discuterons les résultats obtenus (section 4), avant de conclure sur les leçons à tirer de la crise de 2008 au regard de la situation actuelle (conclusion).

\section{$-2-$}

\section{Définir la résilience économique}

Pour certains auteurs, ce seraient la sévérité de la crise de 2008 et l'hétérogénéité des impacts spatiaux qui auraient contribué à populariser le concept de résilience en économie (Giannakis et Bruggeman, 2017). Et, en effet, on compte moins de 10 occurrences par an de la notion en économie avant 2007 sur Web of Science. Le terme de résilience est en revanche plus ancien dans des disciplines comme l'écologie, la mécanique, la psychologie, ou bien encore la géographie physique.

\subsection{La résilience économique, un concept pluridimensionnel}

En 2007, Pendall et al. (2007) proposent une revue de littérature du concept basée sur différents champs disciplinaires (écologie, psychologie, économie, géographie, science politique et archéologie) et soulignent alors l'intérêt de la notion pour comprendre les dynamiques économiques régionales (Hassink, 2010). Le concept est mobilisé pour essayer de comprendre

1 Travail issu du programme POPSU-Métropole, financé par le PUCA et la Métropole Grenoble-Alpes. 
comment les économies régionales ou locales réagissent et récupèrent face aux chocs (Hamdouch et al., 2012 ; Crescenzi et al., 2016).

Martin (2012) identifie quatre dimensions de la résilience : (i) la résistance, qui mesure la sensibilité en termes de revenu régional ou d'emplois face à un choc exogène ; (ii) la récupération qui mesure la rapidité de retour à l'équilibre ; (iii) la réorientation qui indique comment la région change après un choc en modifiant par exemple sa composition sectorielle, et (iv) le renouveau qui est la capacité d'une économie à renouveler son sentier de croissance. Ces quatre dimensions ont été reprises par plusieurs auteurs pour analyser l'impact de la crise de 2008 sur les pays ou régions européennes (Martin et Sunley, 2015 ; Diodato et Weterings, 2015 ; Manca et al., 2017).

Ainsi, deux aspects imbriqués de la résilience sont considérés : l'un en rapport avec le choc et le retour à l'équilibre (on mesure quantitativement l'impact de la crise, le temps mis pour revenir à l'équilibre et les facteurs explicatifs de ces variations) ; l'autre en rapport avec les trajectoires de changement opérées par les territoires pour faire face à la crise (soit à minima en termes de résistance ou bien de réorientation, voire de renouvèlement complet de son sentier de dépendance).

La résilience économique désigne donc la capacité d'une économie locale à surmonter un choc (premier aspect), en absorbant, en s'adaptant ou en se transformant profondément (deuxième aspect).

L'article se concentre sur le premier aspect de la résilience, en calculant, à une échelle fine, l'intensité du choc, puis celle du rebond, avant de définir les temporalités différenciées de retour à l'équilibre. Ces différents éléments composent le cycle de résilience.

\subsection{Le cycle de résilience économique}

Le cycle de résilience désigne une période comprise entre deux crises ou chocs économiques, mesurés par une diminution de la variable observée (le plus souvent l'emploi ou la production). La datation des différents points de retournement observés durant le cycle de résilience permet de mesurer l'intensité de la résilience entre deux points (variation à la baisse puis à la hausse de la variable observée), mais également la temporalité des sous-périodes.

Le calcul des cycles de résilience des EPCI sera basée sur l'analyse des logarithmes naturels de l'emploi privé $(E)$, permettant de lisser la distribution des données. Pour chaque EPCI $(m)$, on détermine les points de retournement entre les phases de récession $(r)$ et d'expansion $(e)^{2}$, comme suit (Figure 1) :

- $P l_{m}$ indique le pic de l'observation avant un premier déclin ;

- $C_{m}$ apparaît après le pic $P l_{m}$ et indique le creux de l'observation avant une première croissance ;

- $R_{m}$ apparaît après le creux $C_{m}$ et désigne le point de récupération, c'est-à-dire lorsque le niveau d'emploi de l'EPCI est revenu à son pic $P 1_{m}$ d'avant le choc ;

\footnotetext{
${ }^{2}$ Les points de retournement sont parfois inexistants, inconnus ou soumis à une condition supplémentaire selon la situation spécifique de chaque EPCI. Les cas particuliers sont les suivants : Lorsqu'il n'y a qu'une seule phase d'expansion $(e)$, il n'y a aucun point de retournement. Lorsque le pic $P I_{m}$ a lieu en 2016 , les points de retournements suivants sont inconnus sur la période analysée, mais le creux $C_{m}$ est daté en 2017 pour assurer la faisabilité des calculs. Lorsqu'il n'y a qu'une seule phase de récession $(r)$, il n'y a aucun point de retournement après le pic $P 1_{m}$. Lorsque le niveau d'emploi de l'EPCI n'est pas revenu à son pic d'avant le choc $P 1_{m}$, il n'y a pas de point de récupération $R_{m}$; et le pic $P 2_{m}$ est daté après le creux $C_{m}$. Lorsqu'aucun déclin ne vient à partir du point $R_{m}-$ ou après le creux $C_{m}$ s'il n'y a pas de récupération -, le pic $P 2_{m}$ est inconnu, mais est daté en 2017 pour assurer la faisabilité des calculs.
} 
- $P 2_{m}$ apparaît à partir du point $R_{m}$ - ou après le creux $C_{m}$ s'il n'y a pas de récupération - et marque la fin du cycle, soit avant un prochain déclin.

On désigne par $t_{P 1}, t_{C}, t_{R}$ et $t_{P 2}$, les années où les points respectifs $P 1_{m}, C_{m}, R_{m}$ et $P 2_{m}$ paraissent.

Figure 1. Cycle de résilience d'un EPCI $(m)$

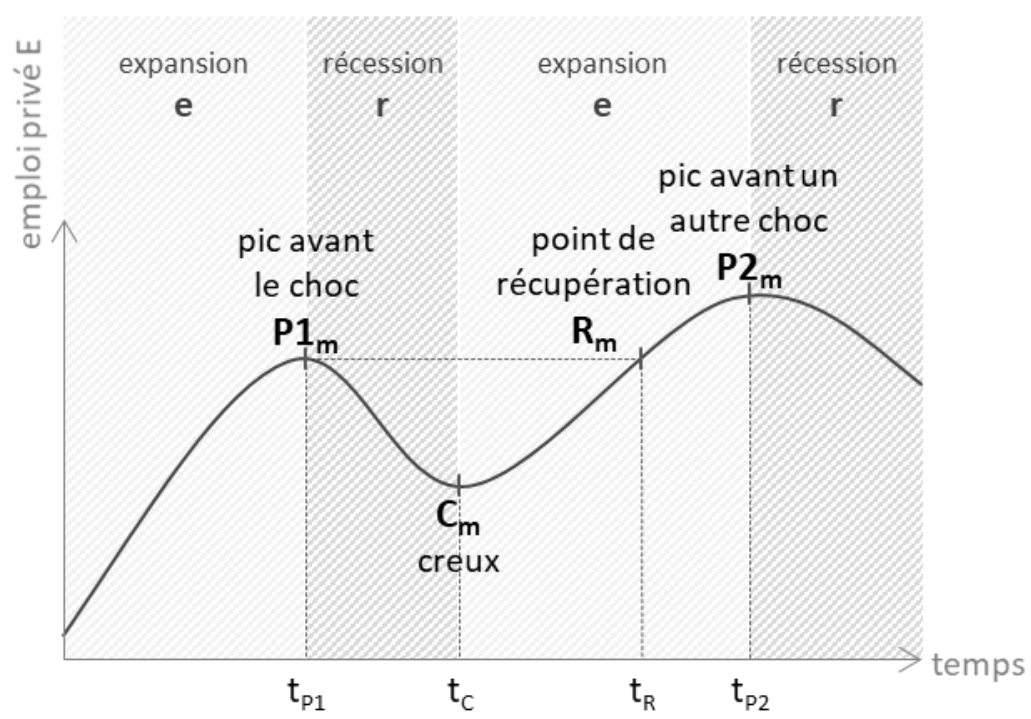

Source : Auteurs.

Avant le pic $P 1_{m}$, l'EPCI n'a pas encore subi de choc, c'est une phase croissante de pré-cycle. Le cycle commence au pic $P 1_{m}$, lorsque l'EPCI subit un premier choc, puis s'ensuit une phase de récession $(r)$. S'il n'y a aucun pic $P l_{m}$, l'économie de l'EPCI est en croissance sur toute la période étudiée. Au creux $C_{m}$, l'EPCI rebondit, c'est le début de la phase d'expansion $(e)$. Au point $R_{m}$, l'EPCI a récupéré son niveau d'avant la crise, son économie continue de croître jusqu'au pic $P 2_{m}$. Lorsque le point $R_{m}$ et le pic $P 2_{m}$ ont lieu la même année, l'EPCI est en déclin juste après avoir récupéré. Au pic $P 2_{m}$, l'EPCI a subi un autre choc, c'est la fin de la phase d'expansion (e) et du cycle. Après le pic $P 2{ }_{m}$, l'économie de l'EPCI n'est plus analysée, c'est une phase post-cycle.

Une fois les cycles de résilience définis, se pose la question des facteurs explicatifs de la résilience.

\subsection{Les facteurs de résilience ou l'avantage métropolitain}

Trois grands ensembles de facteurs de résilience sont régulièrement admis pour qualifier la résilience économique des territoires.

Le premier ensemble de facteurs correspond aux facteurs compositionnels. Il s'agit de la structure ou composition sectorielle de l'économie locale, c'est-à-dire le mélange particulier d'activités économiques et le degré d'interdépendance entre elles (Dissart, 2003). Selon Martin (2012), une structure économique diversifiée est plus résiliente qu'une structure plus spécialisée, car les divers secteurs ont des sensibilités différentes aux changements des entreprises, des 
marchés d'exportation, des conditions monétaires (taux de change et taux d'intérêt), etc. (Brown et Greenbaum, 2016 ; Crescenzi et al., 2016). À l'inverse, si une économie est hautement spécialisée, sa résilience dépend de la nature de sa spécialisation. Les industries de la fabrication et de la construction (activités productives ou manufacturières) sont plus sensibles aux récessions économiques que les services du secteur privé (y compris financiers, commerciaux et d'assurance), eux-mêmes souvent plus sensibles que les services du secteur public (Giannakis et Bruggeman, 2015 ; Martin, 2012). Le tourisme international (hôtellerie et restauration) fut également un secteur résilient dans le contexte de la crise économique de 2008 (Giannakis et Bruggeman, 2015 ; Psycharis et al., 2014). Une autre spécialisation avantageuse en termes de résilience semble être dans la " nouvelle économie », c'est-à-dire les secteurs innovants et à hauts salaires, tels que la fabrication de haute technologie, les services créatifs, médiatiques, numériques, financiers et autres services aux entreprises (Martin et Sunley, 2014 ; Storper et Scott, 2009).

Le second ensemble de facteurs de résilience se rapporte aux facteurs collectifs. Ce sont les caractéristiques et relations entre les entreprises et marchés du travail locaux qui font émerger des cultures et des environnements économiques particuliers (Martin et Sunley, 2014). Deux principaux déterminants de cette compétitivité locale - et ainsi de la capacité locale à réagir et à s'adapter à un choc - sont le capital humain et les efforts de compétences et d'innovation. Le capital humain serait positivement associé aux performances économiques pendant un choc, contrairement à une forte intensité de recherche et développement (R\&D). Par conséquent, ce n'est pas l'innovation axée sur la technologie (représentée par les investissements de R\&D) qui serait une clé de la résistance économique, mais plutôt un environnement propice à l'innovation, reflété par l'abondance de main-d'œuvre qualifiée (Crescenzi et al., 2016 ; Crescenzi et Rodríguez-Pose, 2011 ; Di Caro, 2015). Les flux de capital humain et les mouvements migratoires ont alors un rôle important, dans la mesure où ils contribuent positivement au développement économique par une demande accrue de biens et services et ainsi une dépense supplémentaire de revenus (Danson, 2002 ; Faggian et McCann, 2009 ; Giannakis et Bruggeman, 2017). De même, l'accessibilité et la connectivité spatiale facilitent les flux de connaissances entre les personnes et améliorent l'efficacité des marchés du travail locaux (Giannakis et Bruggeman, 2017 ; Östh et al., 2015).

Le troisième ensemble de facteurs de résistance aux chocs concerne les facteurs contextuels ou facteurs macroéconomiques de transmission de la crise. Ce sont non seulement les contextes locaux institutionnel et de gouvernance, mais aussi les déséquilibres macro-nationaux. On peut noter par exemple comme facteurs aggravant, l'important déficit de la balance courante ou une grande ouverture au commerce (ex. exportations considérables vers les marchés émergents, solde net très positif d'investissements directs étrangers) (Crescenzi et al., 2016 ; Groot et al., 2011 ; Lane et Milesi-Ferretti, 2010). Les fortes connectivités aux marchés internationaux se retrouvent généralement dans les grandes villes ou zones métropolitaines et sont souvent sujettes à l'effondrement (Dijkstra et al., 2015 ; Donald et al., 2014 ; Psycharis et al., 2014).

Ce troisième facteur indique que les territoires ayant un degré d'urbanisation élevé subissent les conséquences les plus immédiates d'un choc. En revanche, les deux premiers facteurs expliquent leur capacité à s'adapter très rapidement (Fratesi et Perucca, 2018). 


\section{Méthodologie pour mesurer la résilience économique d'un territoire}

Le choix des variables et de l'échelle d'analyse est conditionné par nos hypothèses de travail. Nous souhaitons tirer des enseignements de la crise de 2008 en termes de résilience des métropoles, au regard des autres territoires, et de l'éventuel impact sur les fractures territoriales. Il est donc important de travailler à une échelle suffisamment fine pour distinguer les dynamiques des métropoles de celles de leur périphérie. Par ailleurs, nous souhaitons mener une analyse de longue période afin de révéler les cycles de résilience. Il nous faut donc mobiliser une base de données annuelles et mettre en place des méthodes de calcul qui permettent de révéler les intensités et les temporalités de la résilience économique.

\subsection{Variable et échelle d'analyse}

Les deux variables utilisées pour mesurer la résilience économique d'une région ou d'un territoire sont la production, mesurée avec le PIB, et l'emploi (Bristow et Healy, 2018 ; Eraydin, 2016). Outre la disponibilité des données à une échelle fine d'analyse, les variations de l'emploi reflètent mieux l'impact social du choc que la production (Fratesi et Rodríguez-Pose, 2016). Par ailleurs, l'emploi a tendance à revenir aux niveaux d'avant la crise avec un décalage plus long que la production. Les conséquences des graves crises économiques passées ont révélé que le cycle de résilience mesuré en emplois dure 4,8 ans, alors que celui de la production ne dure que 1,9 ans (Reinhart et Rogoff, 2009). Comme d'autres travaux (Brakman et al., 2015 ; Crescenzi et al., 2016 ; Giannakis et Bruggeman, 2015), nous privilégions l'emploi pour étudier la résilience économique des territoires français.

En France, nous connaissons le nombre d'emplois salariés privés annuel par secteur d'activité depuis 2007 à l'échelle communale. Afin de tenir compte des emplois non-salariés, particulièrement impactées par la crise économique (à l'image des chefs d'entreprises, des autoentrepreneurs, des artisans) ces données sont complétées par celles du Répertoire des Entreprises et des Établissements de l'Insee. Au total, pour l'ensemble de l'emploi privé étudié (salarié et non-salarié), nous obtenons 20,7 millions d'emplois renseignés à l'échelle des 35000 communes.

Notre analyse porte sur la capacité de résilience économique des territoires, mesurée en termes de variation des emplois du secteur privé (salarié et non salarié). Ces emplois, qui couvrent près de $79 \%$ de l'emploi total français en 2019, sont ceux qui ont subi les plus fortes pertes en 2008. En effet, entre 2007 et 2009, l'Insee note que l'emploi public a augmenté de $+0,4 \%$. Par ailleurs, l'emploi public n'est plus renseigné en France à l'échelle locale depuis quelques années et ne pourrait donc pas être étudié avec la même finesse d'analyse.

L'échelle d'analyse retenue est celle des Etablissements Publics de Coopération Intercommunale (EPCI), qui couvrent l'ensemble du territoire national. Ce choix nous permet de comparer la situation des métropoles institutionnelles à celle des autres intercommunalités proches ou éloignées. De plus, si les régions demeurent les chefs de file du développement économique et de l'aménagement du territoire en France, les EPCI, et notamment les métropoles, sont devenus des périmètres d'action essentiels en termes de développement économique local. Une analyse à l'échelle des zones d'emplois - très utilisée en économie régionale - nous éloignerait de la question posée, dans la mesure où ces zones sont très étendues et recouvrent des dynamiques urbaines et rurales. Pour pallier ces limites, l'Insee prévoit d'ailleurs de revoir 
complètement ce zonage statistique afin de mieux distinguer les zones urbaines et périurbaines, des espaces extra-métropolitains. Par exemple, les zones d'emplois de Toulouse ou de Grenoble dépassent largement leur zone réelle d'influence, y compris mesurée en termes de flux domiciletravail.

La variation des emplois du secteur privé de 2007 à 2017 est donc analysée à l'échelle des 1236 EPCI français. Les 20 millions d'emplois privés se répartissent entre les métropoles, à hauteur de $41 \%$, les communautés d'agglomération (32\%), les communautés de communes (23 $\%)$ et les communautés urbaines $(5 \%)$ (Tableau 1).

Tableau 1. Nombre et composition des EPCI de France métropolitaine en 2019 par type et par tranche de population totale (2016).

\begin{tabular}{|c|c|c|c|c|c|}
\hline Ensemble des EPCI & $\begin{array}{c}\text { Nombre } \\
\text { d'EPCI }\end{array}$ & $\begin{array}{c}\text { Nombre moyen } \\
\text { de communes } \\
\text { par EPCI }\end{array}$ & $\begin{array}{c}\text { Population } \\
\text { moyenne } \\
\mathbf{( 2 0 1 6 )}\end{array}$ & $\begin{array}{c}\text { Nombre moyen } \\
\text { d'emplois privés } \\
(\mathbf{2 0 1 6})\end{array}$ & $\begin{array}{c}\text { Répartition } \\
\text { des emplois } \\
\text { privés (2016) }\end{array}$ \\
\hline $\begin{array}{c}\text { Communautés de } \\
\text { Communes }\end{array}$ & 993 & 26 & $\mathbf{5 3 2 5 9}$ & $\mathbf{1 6 3 0 0}$ & $\mathbf{1 0 0 \%}$ \\
\hline $\begin{array}{c}\text { Communautés } \\
\text { d'Agglomération }\end{array}$ & 208 & 36 & 104090 & 30574 & $32 \%$ \\
\hline $\begin{array}{c}\text { Communautés } \\
\text { Urbaines }\end{array}$ & 13 & 49 & 224845 & 70748 & $5 \%$ \\
\hline $\begin{array}{c}\text { Métropoles de droit } \\
\text { commun }\end{array}$ & 19 & 45 & 473591 & 179139 & $17 \%$ \\
\hline $\begin{array}{c}\text { Métropoles à statut } \\
\text { particulier }\end{array}$ & 2 & 28 & 4494521 & 2098856 & $21 \%$ \\
\hline Métropole de Lyon & 1 & 81 & 1400134 & 612927 & $3 \%$ \\
\hline
\end{tabular}

Sources : IGN 2019 ; Insee, Recensement de la population 2016 ; Acoss \& REE-Insee 2016, calcul des auteurs.

La variable d'observation et l'échelle étant définies, voyons à présent les méthodes de calcul proposées.

\subsection{Intensité de la résilience}

Pour calculer les intensités de résilience, nous distinguons $E_{P 1_{m}}, E_{C_{m}}$ et $E_{P 2_{m}}$ comme étant respectivement, les nombres d'emplois privés au pic $P 1_{m}$, au creux $C_{m}$ et au pic $P 2_{m}$.

- $I_{m}^{r}$ désigne le taux de déclin économique - ou l'intensité de résistance - de l'EPCI, c'està-dire son pourcentage de perte d'emplois privés. Il est calculé entre les points $P 1_{m}$ et $C_{m}$ (phase de récession $(r)$ ), comme suit :

$$
I_{m}^{r}=\frac{E_{C_{m}}-E_{P 1_{m}}}{E_{P 1_{m}}}
$$

- $\quad I_{m}^{e}$ désigne le taux de croissance économique - ou l'intensité de reprise - de l'EPCI, c'est-à-dire son pourcentage de gain d'emplois privés. Il est calculé entre les points $C_{m}$ et $P 2_{m}$ (phase d'expansion $(e)$ ), comme suit : 


$$
I_{m}^{e}=\frac{E_{P 2_{m}}-E_{C_{m}}}{E_{C_{m}}}
$$

Figure 2. Intensités et temporalités des variations d'emploi privé

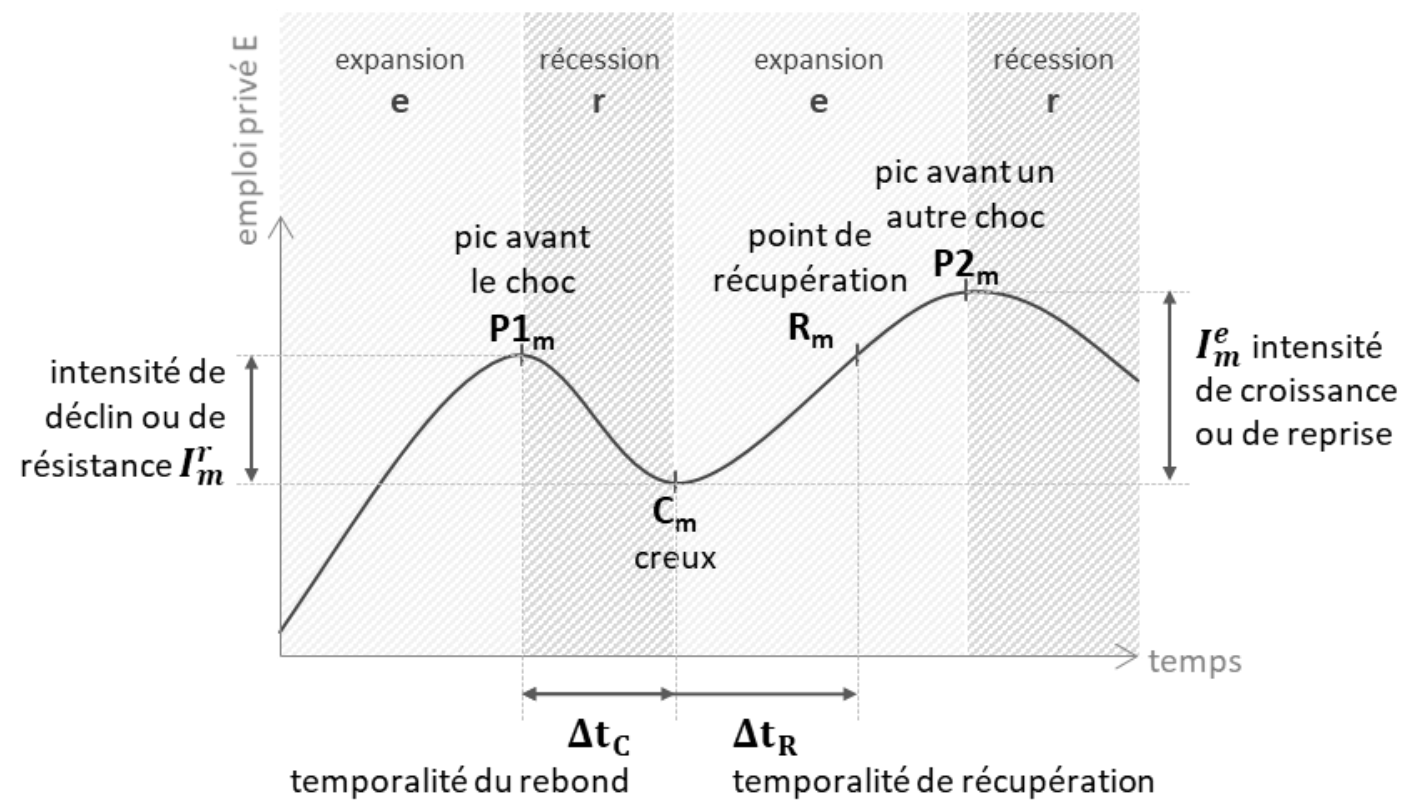

Source : Auteurs.

À partir des deux variables d'intensité de la résilience présentées ci-dessus, il est possible de construire un indicateur de résilience à deux dimensions, sur le même modèle que celui proposé par Martin et al. (2016).

Deux indices sont définis :

- L'indice de sensibilité (SI) correspond au taux de déclin de l'emploi privé des EPCI $\left(\Delta E_{m}^{r}\right)$ par rapport au recul moyen national $\left(\Delta E_{n}^{r}\right)$ pendant la crise, soit :

$$
S I_{m}=\frac{\Delta E_{m}^{r}-\Delta E_{n}^{r}}{\left|\Delta E_{n}^{r}\right|}
$$

- L'indice de récupération (RI) est défini comme le taux de croissance de l'emploi privé des EPCI $\left(\Delta E_{m}^{e}\right)$ par rapport à l'expansion moyenne du pays $\left(\Delta E_{n}^{e}\right)$ après la crise, soit :

$$
R I_{m}=\frac{\Delta E_{m}^{e}-\Delta E_{n}^{e}}{\left|\Delta E_{n}^{e}\right|}
$$

Une valeur positive de l'indice de sensibilité (SI) indique qu'un EPCI est plus résistant à la récession que la moyenne nationale, une valeur négative qu'il est donc moins résistant. Similairement, un EPCI qui a une valeur positive de l'indice de récupération (RI) a une capacité de reprise supérieure à la moyenne, et inversement s'il a une valeur négative.

Ces mesures d'intensité d'abord en termes de déclin, puis en termes de reprise, sont importantes pour qualifier l'ampleur du choc. Pour autant, une autre dimension de la résilience est fondamentale et plus rarement étudiée. Il s'agit de la temporalité du cycle. 


\subsection{Temporalité de la résilience}

Nous pouvons ainsi calculer deux temporalités ou durées pour caractériser la résilience :

- La durée du déclin $\Delta t_{C}$ est mesurée en années entre le premier pic $P 1_{m}$ et le creux $C_{m}$, avec :

$$
\Delta t_{C}=t_{C}-t_{P 1}
$$

Le rebond peut être qualifié de rapide lorsque la durée du déclin calculée pour chaque intercommunalité est inférieure ou égale à celle du cycle de référence ( 1 an pour la moyenne des EPCI et 2 ans pour la moyenne des métropoles);

- La durée nécessaire à l'EPCI pour revenir à son pic d'avant le choc $\Delta t_{R}$ est mesurée en années entre le premier pic $P l_{m}$ et le point de récupération $R_{m}$, soit :

$$
\Delta t_{R}=t_{R}-t_{P 1}
$$

La récupération sera qualifiée de rapide, lorsque cette durée est inférieure ou égale à celle du cycle de référence ( 2 ans pour la moyenne des EPCI et 3 ans pour la moyenne des métropoles), sinon elle est lente.

Nous pouvons à présent calculer le cycle de résilience de chaque EPCI français, à la fois en termes d'intensité et de temporalité.

\section{$-4-$ \\ Analyse de la résilience économique des territoires}

Les résultats présentés ici permettent de spécifier les cycles économiques des métropoles et des autres EPCI entre 2007 et 2017. Nous verrons que l'intensité et la temporalité de la résilience se déclinent différemment selon les territoires. La question est de savoir s'il existe en la matière une éventuelle spécificité métropolitaine qui accroitrait les inégalités économiques.

\subsection{Des cycles économiques différenciés}

La Figure 3 nous montre, qu'en moyenne, les EPCI français ont débuté leur récession en 2008, puis rebondi en 2009 et récupéré leur niveau d'emploi en 2010, avant de subir un deuxième choc - plus léger - en 2011.

En comparaison, les métropoles ont enregistré, en moyenne, une perte d'emplois dès 2007, avec cependant un taux de déclin $(-1,06 \%)$ légèrement moins intense que celui des EPCI $(-1,15$ $\%)$. De même que les EPCI en moyenne, les métropoles rebondissent en 2009 et récupèrent en 2010. Toutefois, elles ne subissent pas d'autres chocs ultérieurement, et enregistrent alors un taux de croissance des emplois sur la fin de période $(+10,87 \%)$ beaucoup plus intense que celui des $\operatorname{EPCI}(+2,05 \%)$. 
Figure 3. Cycle de résilience des EPCI et des métropoles

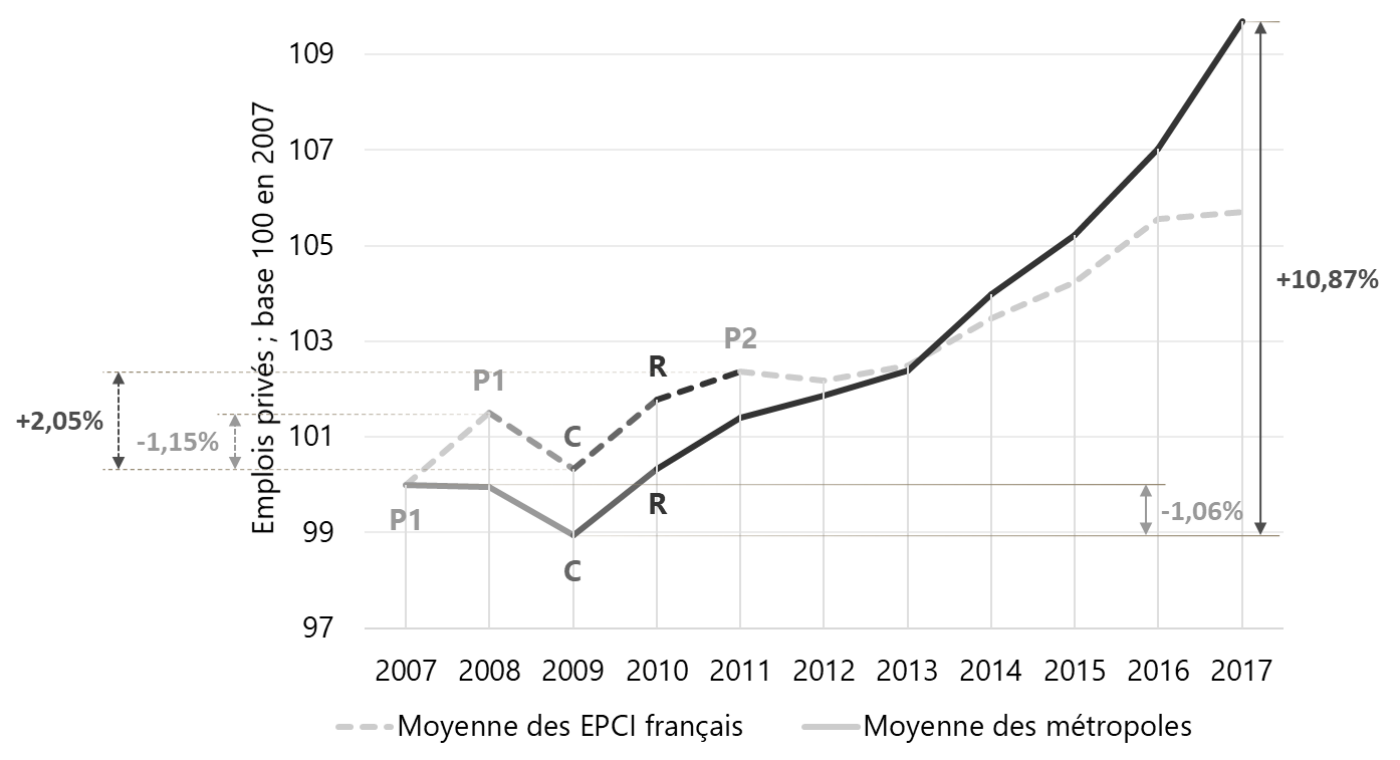

Source : Auteurs, Acoss et REE-Insee.

Ces éléments semblent donc aller dans le sens d'une meilleure résilience des métropoles. Plus précisément, les métropoles ne sont pas plus résistantes au déclin (les pertes d'emplois sont aussi importantes qu'ailleurs), ni plus performantes en termes de temporalité du rebond (la reprise s'observe dans les deux cas en 2009), mais en revanche plus avantagées en termes d'intensité de reprise. Pour autant, on sait aussi que ce type de moyennes masque d'importantes différences géographiques. Les métropoles de Nantes, Grenoble, Rouen... n'ont, par exemple, pas le même cycle de résilience (Tableau 2 en annexe). Une approche plus territorialisée de la question peut donc s'avérer pertinente.

\subsection{Mesurer l'intensité de la résilience}

Tout d'abord, voyons quelles ont été les intensités de pertes et de gains d'emplois selon les territoires.

Les communautés de communes de moins de 25000 habitants enregistrent les plus fortes pertes d'emploi privé (-4,71\%), avec néanmoins un écart-type plus important que dans les autres strates ce qui rend compte aussi de la variabilité des situations dans cette catégorie. Ces mêmes EPCI comptent une croissance de $+4,27 \%$ après la crise, ce qui les rapproche cette fois de la moyenne française (Tableau 3 en annexe). À l'opposé, la catégorie d'EPCI à la croissance la plus intense est celle des communautés de communes de plus de 25000 habitants, ainsi que celle des métropoles de plus de 450000 habitants, soit des types de villes très différentes. Ces chiffres montrent à quel point le type ou la taille de l'intercommunalité a peu joué dans la perte ou le gain d'emplois pendant la crise, confortant les analyses et propos de Bouba-Olga et Grossetti (2015).

En revanche, ce qui apparait sur la Carte 1, c'est le fait que l'on observe une géographie régionale de ces processus, plutôt qu'une fracture entre centre et périphérie ou bien encore entre métropoles et reste du territoire. Ainsi, on constate une concentration de forts taux de déclin dans 
les régions nord du Centre-Val de Loire, de la Bourgogne-Franche-Comté, du Grand Est, de la Normandie et des Hauts-de-France. Ces cinq régions regroupent $69 \%$ des EPCI dont le taux de déclin est inférieur de plus de $4 \%$ à la moyenne française. Au sein de ces régions seule la métropole de Lille résiste mieux en moyenne que les autres EPCI de la région (cf. détail en Tableau 4 en annexe). Globalement, les métropoles ont enregistré d'importantes pertes d'emplois dès le début de la crise. Seulement cinq métropoles ont connu un plus faible taux de perte d'emplois que les autres EPCI de leur région respective (Lyon, Clermont-Ferrand, Lille, Bordeaux, Nantes). Treize métropoles ont, au contraire, enregistré un plus fort déclin. Enfin, Toulouse, Aix-Marseille, Montpellier et Toulon n'ont pas connu de récession pendant la crise. Ces quatre métropoles du Sud de la France ont pu profiter des dynamiques résidentielles et touristiques. En effet, on sait que les régions européennes résidentielles attractives, mieux dotées en biens collectifs (stock de capital naturel et culturel), ont été plus résilientes, car dans la phase initiale de la crise de 2008, la demande des consommateurs en équipements a moins diminué que pour les autres éléments du capital territorial (Fratesi et Perucca, 2018). De même, les régions grecques spécialisées dans les activités de tourisme international (hôtellerie et restauration) ont mieux résisté à la crise (Psycharis et al., 2014 ; Giannakis et Bruggeman, 2015).

L'analyse du premier cycle de résilience en termes d'intensité des pertes d'emplois subie souligne donc une fracture régionale, marquée par des facteurs structurels, bien plus qu'une réelle divergence entre métropoles et reste du territoire.

Carte 1. Déclin des emplois privés (en \%) entre les pics P1m et les creux $\mathrm{Cm}$
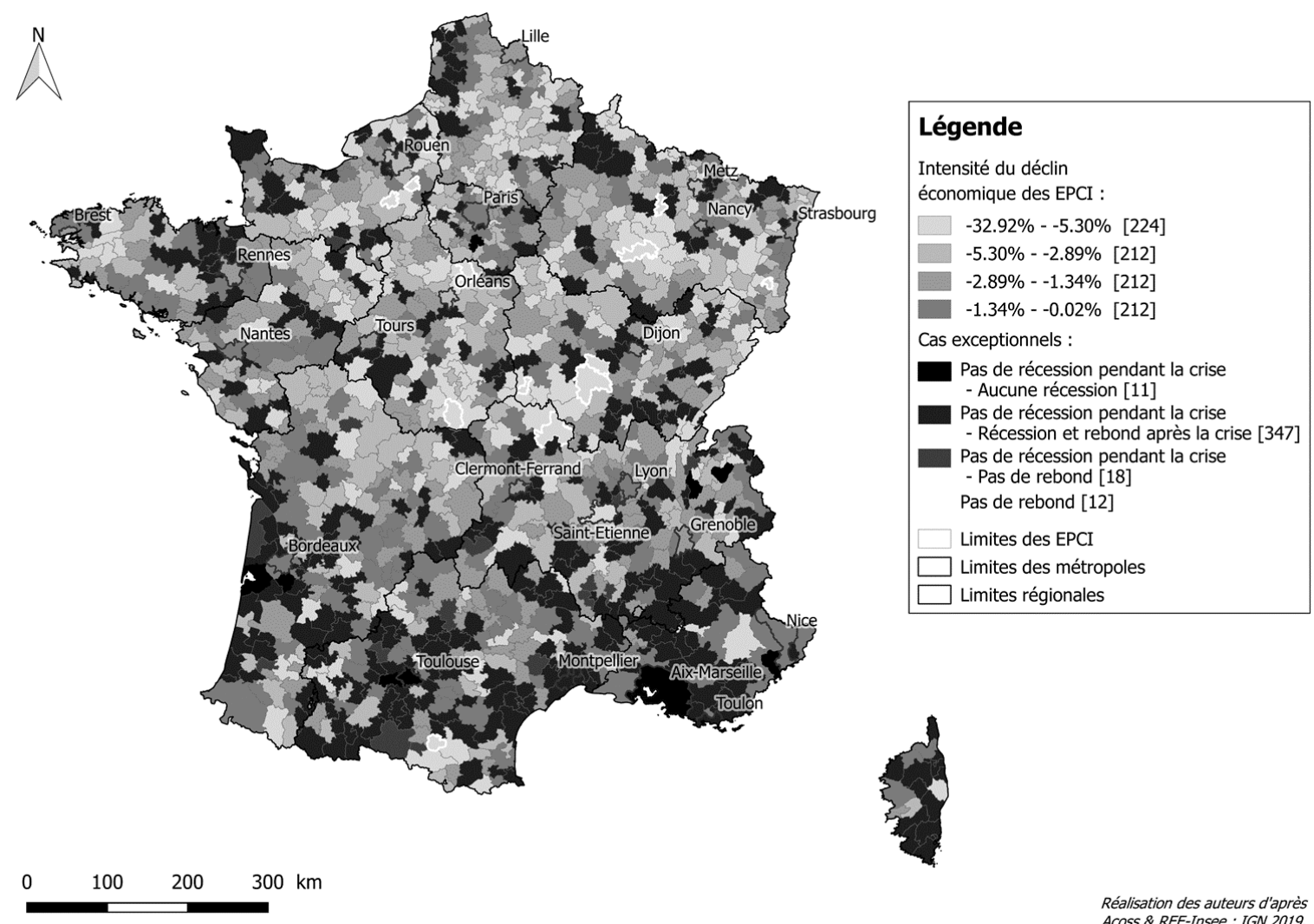
Réalisation des auteurs d'après
Acoss \& REE-Insee ; IGN 2019. 
En revanche, les disparités régionales en termes de reprise économique sont moins nettes (Carte 2). On observe des taux de croissance forte dans les EPCI des régions du Sud telles que la Corse, le sud d'Auvergne-Rhône-Alpes et Provence-Alpes-Côte d'Azur, mais aussi dans des régions comme l'Île-de-France ou les Pays de la Loire. Ces taux révèlent également une plus faible croissance ou reprise économique dans ce que les géographes appellent la diagonale du vide, s'étendant du Nord-Est au Sud-Ouest et désignant ainsi des espaces de faibles densités le plus souvent éloignés de grands pôles urbains. Ces régions rurales n'abritent pas de grandes zones urbaines denses à l'économie diversifiée et potentiellement plus résilientes (Glaeser et al., 2014). En effet, on constate, cette fois, une plus forte croissance pour les métropoles. 17 métropoles sur 22 affichent des taux de croissance plus élevés que ceux des autres EPCI de leur région. Les cinq métropoles faisant exception sont Nice, Nancy, Grenoble, Saint-Etienne et Toulon (Tableau 4 en annexe).

Ainsi, bien que les zones métropolitaines soient généralement plus rapidement touchées en période de crise de par leur spécialisation tertiaire (on voit que les emplois ont diminué dès 2007 dans les métropoles), la plupart d'entre elles ont la capacité d'attirer des fonctions de grande valeur, des activités à forte coopération, et développent des projets d'accessibilité de haute qualité. Ce potentiel a ainsi permis aux régions européennes métropolitaines d'être les premières à gagner pendant la période de reprise (Capello et al., 2015).

Carte 2. Croissance des emplois privés (en \%) entre les creux $\mathrm{Cm}$ et les pics $\mathrm{P} 2 \mathrm{~m}$
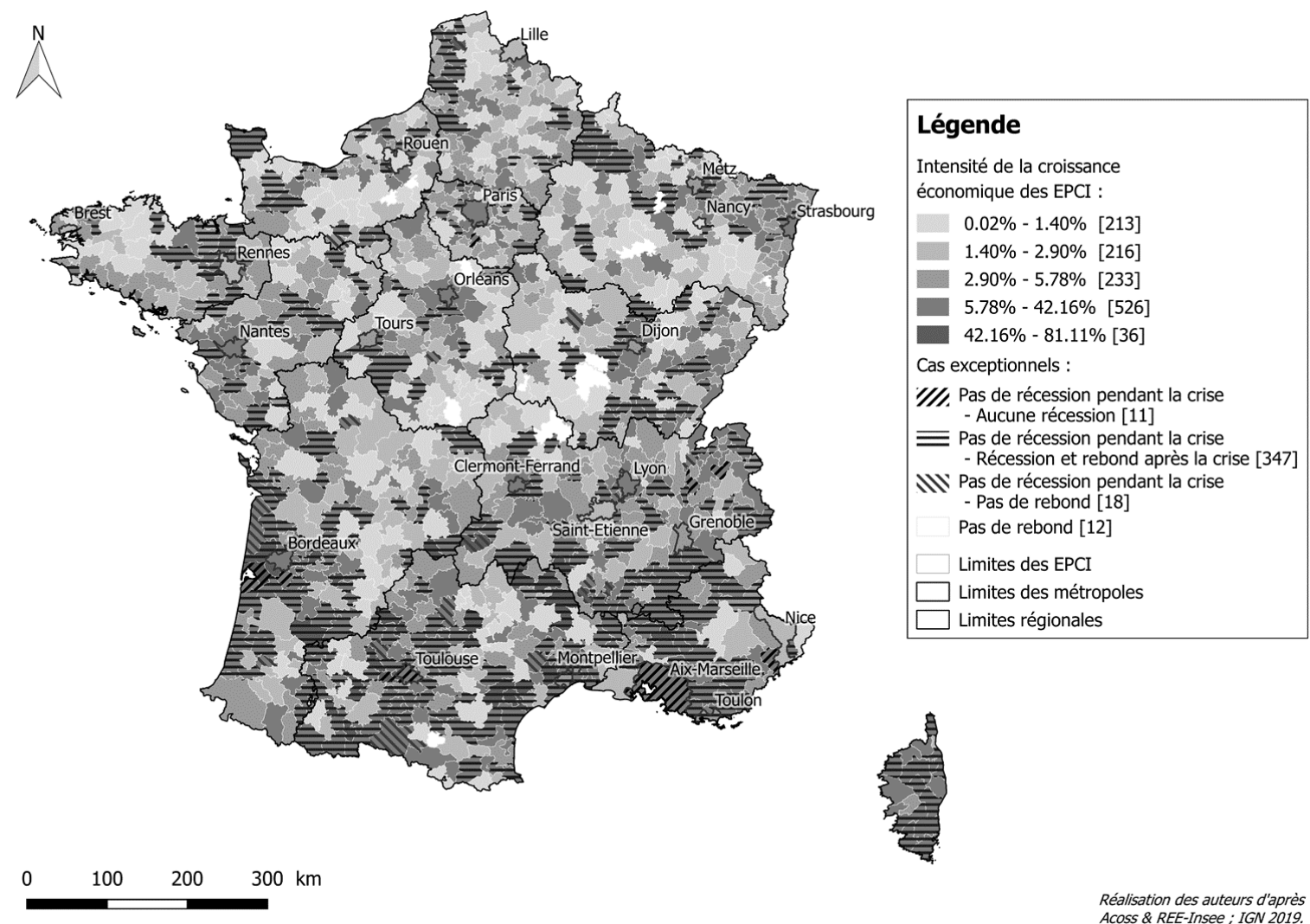

Voyons à présent les résultats cumulés de ces deux dynamiques contenus dans l'indice d'intensité de résilience. 


\subsection{Indice bidimensionnel d'intensité de résilience}

L'indice de sensibilité (SI) mesure la capacité de résistance (ou rebond) d'un EPCI vis-à-vis de la moyenne nationale. Par exemple, SI est égal à 0,48 pour la Métropole Nice Côte d'Azur. Ceci indique que son économie est $48 \%$ plus résistante que la moyenne des intercommunalités. Et la valeur de $-1,18$ pour Grenoble-Alpes-Métropole montre que sa résistance est $118 \%$ inférieure à celle de la moyenne intercommunale.

Parallèlement, l'indice de récupération (RI) mesure la capacité de reprise (ou récupération) par rapport à la moyenne. La valeur de $-0,11$ pour la Métropole du Grand Paris montre que sa résistance est $11 \%$ inférieure à celle de la moyenne intercommunale. Avec une valeur de RI de 1,51, la Métropole du Grand Paris a connu, par contre, une récupération $151 \%$ supérieure à celle de l'économie moyenne intercommunale.

Cela donne donc une configuration en deux dimensions des possibilités de résilience (Figure 4 et Figure 5). Les calculs sont réalisés et discutés à l'échelle des EPCI, puis plus spécifiquement sur le cas des métropoles.

374 EPCI (30 \%) ont une moindre résilience (faibles capacités de rebond et de récupération) et $427 \mathrm{EPCI}$ (35\%) ont un fort rebond (ou bonne résistance), mais une faible récupération (ou mauvaise reprise). Le reste des EPCI se placent quasi-équitablement entre les deux quadrants droits. 231 EPCI $(19 \%)$ ont une meilleure résilience globale (fortes capacités de rebond et de récupération) et $204 \mathrm{EPCI}$ (17\%) ont un faible rebond, mais une forte récupération.

Figure 4. Représentation bidimensionnelle des intensités de résilience des EPCI de France métropolitaine

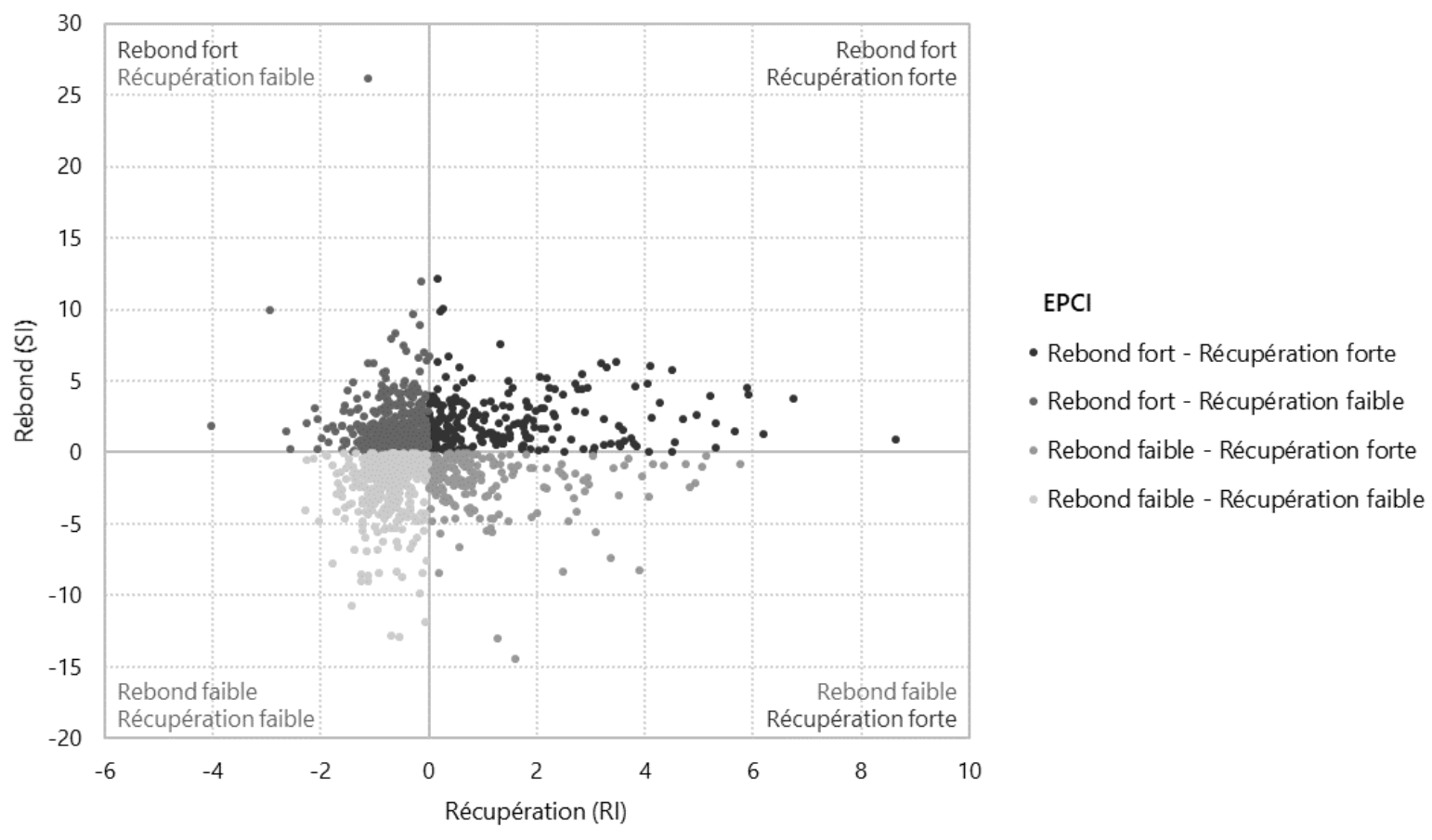

Source : Auteurs, Acoss et REE-Insee. 
Figure 5 Représentation bidimensionnelle des intensités de résilience des métropoles

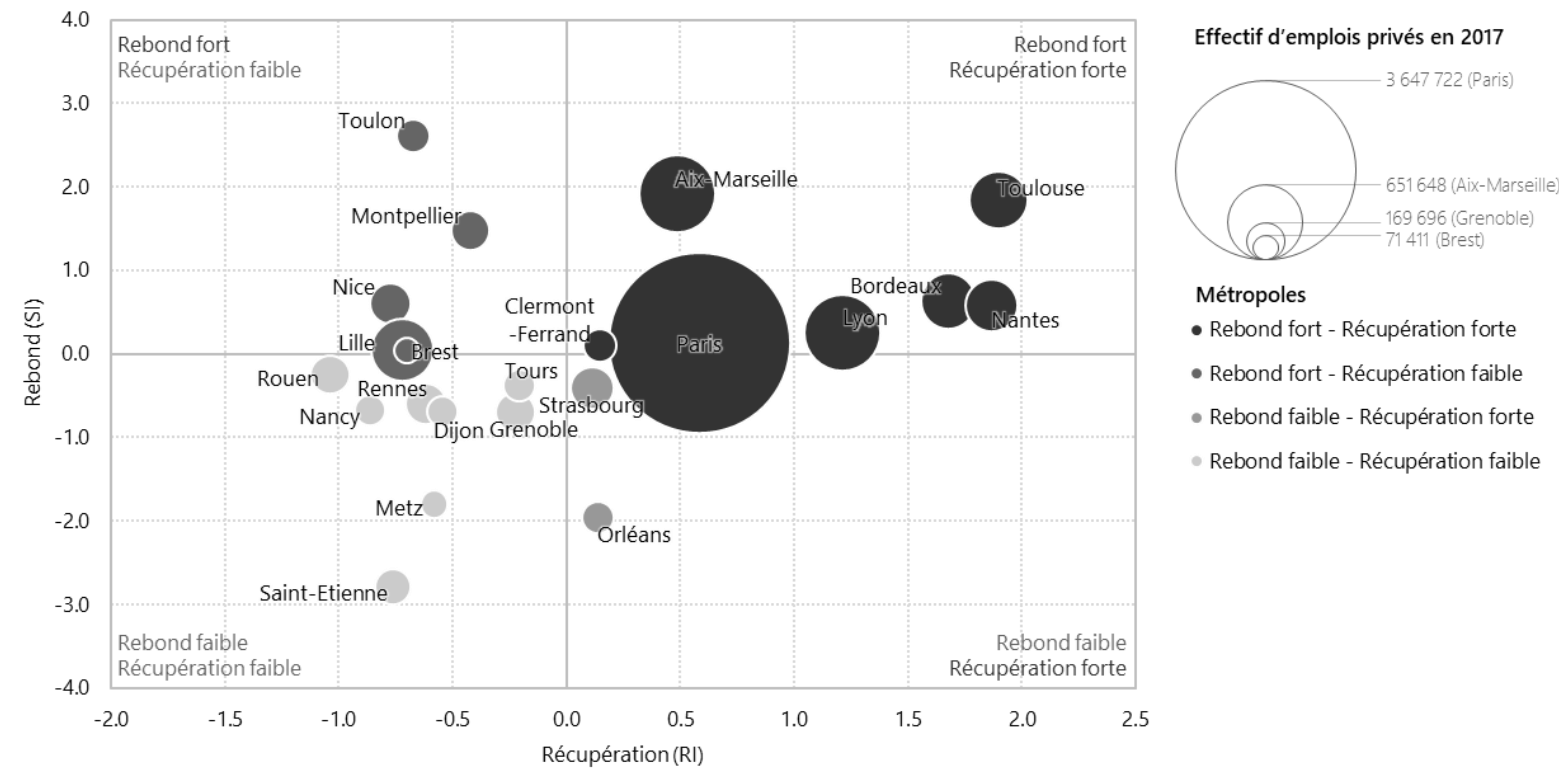

Source : Auteurs, Acoss et REE-Insee.

La géographie de cet indicateur met en évidence les inégalités territoriales en termes de résilience économique (Carte 3). Les régions d'une moitié nord de la France, telles que le Grand Est, le Centre-Val de Loire, les Hauts-de-France, la Bourgogne-Franche-Comté, la Normandie, les Pays de la Loire, l'Île-de-France et la Bretagne, concentrent $74 \%$ des EPCI de moindre résilience, cumulant donc fortes pertes et faibles gains. La majorité des métropoles de ces régions sont dans la même situation que les autres EPCI, à savoir Rouen, Metz, Nancy, Dijon, Brest, Rennes et Lille. Font exception, Paris, Tours, Orléans et Strasbourg qui affichent une mauvaise résistance mais une bonne reprise. Enfin, dans ce contexte régional de faible résilience, seule Nantes finalement cumule bonne résistance et bonne reprise (Figure 11).

Les régions du sud de la France, telles que la Corse, Provence-Alpes-Côte d'Azur, l'Occitanie, la Nouvelle-Aquitaine et l'Auvergne-Rhône-Alpes, concentrent 65 \% des EPCI de meilleure résilience, profil qui concerne également les métropoles de Lyon, Aix-Marseille, Toulouse et Bordeaux. Ces régions comprennent également 3 métropoles de bonne résistance mais mauvaise reprise depuis la crise (Montpellier, Nice et Toulon), 2 métropoles de mauvaise résistance mais bonne reprise (Grenoble et Clermont), et 1 métropole de moindre résilience, celle de SaintEtienne (Figure 11).

Les autres catégories d'EPCI sont réparties de façon plus homogène sur le territoire français, même si l'on peut voir une légère sur-représentation du type "bonne résistance - mauvaise reprise » dans les régions d'une moitié sud et, parallèlement, une légère prédominance des EPCI de mauvaise résistance mais bonne reprise dans les régions plus au nord. 
Carte 3. Typologie des EPCI selon l'indicateur bidimensionnel d'intensité de résilience
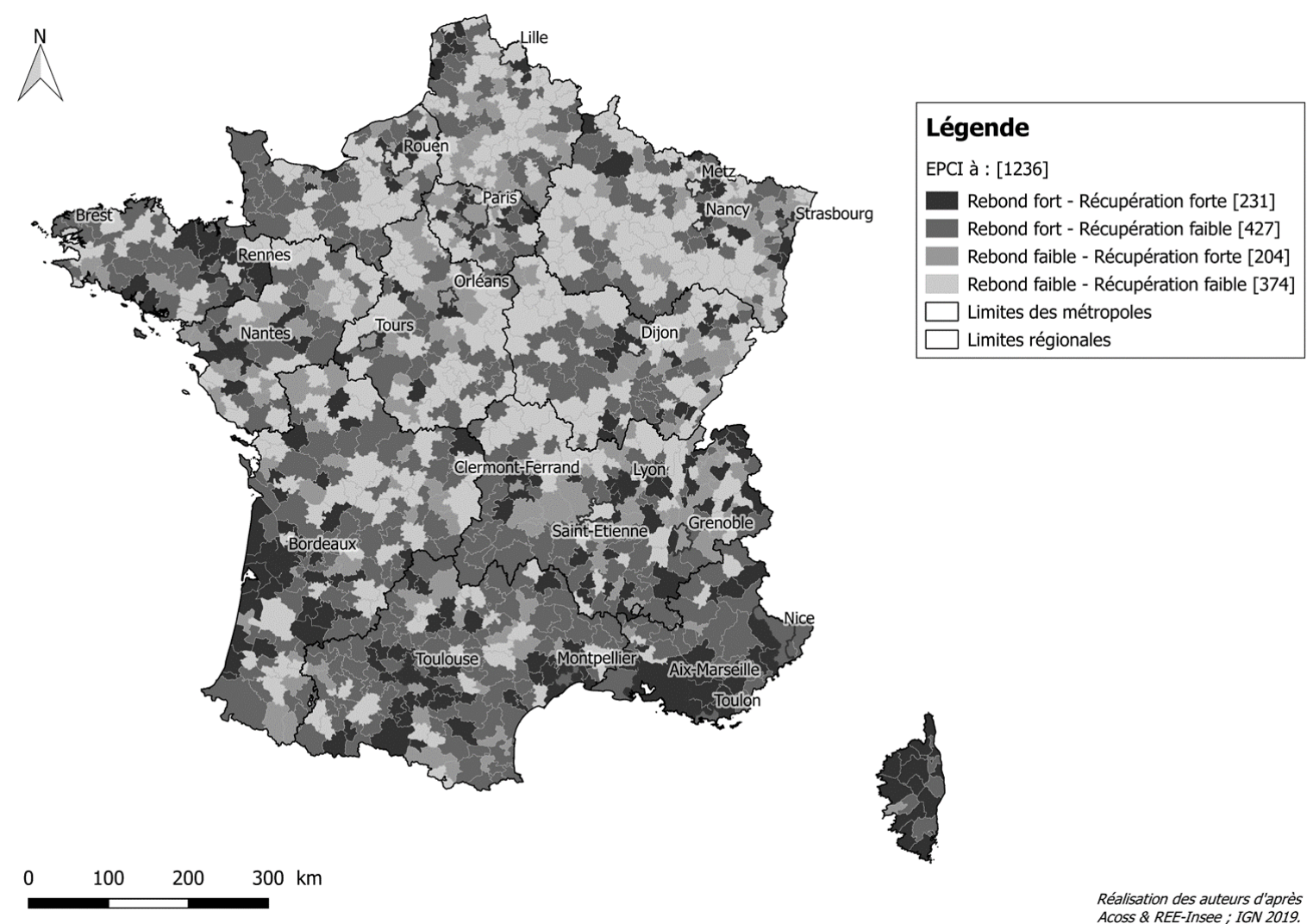
Réalisation des auteurs d'après
Acoss \& REE-Insee ; IGN 2019.

Ces résultats montrent qu'en termes d'intensité de résilience, la crise a plutôt creusé les inégalités entre les régions souvent considérées comme productives ou de tradition industrielle, situées dans une moitié nord du pays, et celles, plus au sud, qui profitent des dynamiques résidentielles et touristiques qui se sont poursuivies malgré la crise de 2008. Finalement, si les atouts métropolitains restent un facteur essentiel de la reprise économique rapide des grandes villes, ils ne génèrent pas de rupture, ou de dynamiques opposées, entre centre et périphérie. C'est donc plutôt en termes de fractures régionales, solidarités locales, que se déclinent les impacts de la crise de 2008, tout au moins en termes d'intensité.

Que nous apprend à présent l'analyse des temporalités de résilience ?

\subsection{Mesurer la temporalité de la résilience}

Concernant la temporalité de la résilience, nous pouvons, d'une part, observer l'année des différents points de retournement du cycle et la durée des différentes phases.

Nous calculons d'abord, chaque année entre 2007 et 2017, le nombre d'EPCI ayant connu son premier déclin, sa première croissance et sa récupération de l'emploi privé. 
$17 \%$ des EPCI ont connu une baisse d'emplois, ou pics $P 1_{m}$, dès $2007^{3}$ et $48 \%$ à partir seulement de 2008 (Figure 6). Pour les métropoles, c'est l'inverse, 12 (soit $55 \%$ ) ont connu un déclin d'emplois en 2007 et $6(27 \%)$ en 2008. Les métropoles subissent la crise avant les autres EPCI.

La répartition des points de creux $C_{m}$ sur la période a également été calculée (Figure 7). Dès 2009, la première expansion des emplois privés se manifeste pour 561 EPCI (hors métropoles) (soit $46 \%$ ). On relève également dès cette année-là un rebond pour 17 métropoles (77\%), dont celles de Grenoble, Nice, Metz, Dijon, Brest, Bordeaux, Rennes, Tours, Saint-Etienne, Nantes, Orléans, Nancy, Lille, Clermont, Strasbourg, Paris et Lyon. La métropole de Rouen ne retrouve cependant une croissance de ses emplois qu'en 2013, soit après 5 années de déclin. On observe que $31 \mathrm{EPCI}$ (soit 2,6 \%) ne connaissent pas de reprise de l'emploi depuis la crise économique.

Enfin, il est intéressant de repérer les dates de récupération $R_{m}$ (Figure 8). Cette fois, et contrairement aux deux points de retournement, on constate une moins grande régularité des situations d'un EPCI à l'autre. En effet, aucune date n'approche les $50 \%$ des cas observés, que ce soit pour les métropoles ou les autres types d'EPCI. Nice, Lyon, Bordeaux, Nantes récupèrent en 2010, Brest, Paris, Rennes, Lille en 2011, Clermont en 2012, Grenoble en 2014, Tours, Strasbourg en 2015, Orléans en 2016, Dijon en 2017. 4 métropoles n'ont toujours pas retrouvé leur niveau d'emplois en 2017 : Rouen, Metz, Saint-Etienne et Nancy. Enfin, pour les autres types d'EPCI, on note que plus d'un tiers (445 exactement) n'ont toujours pas récupéré de la crise de 2008 en 2017.

Figure 6. Nombre d'EPCI connaissant un premier déclin de l'emploi privé par an

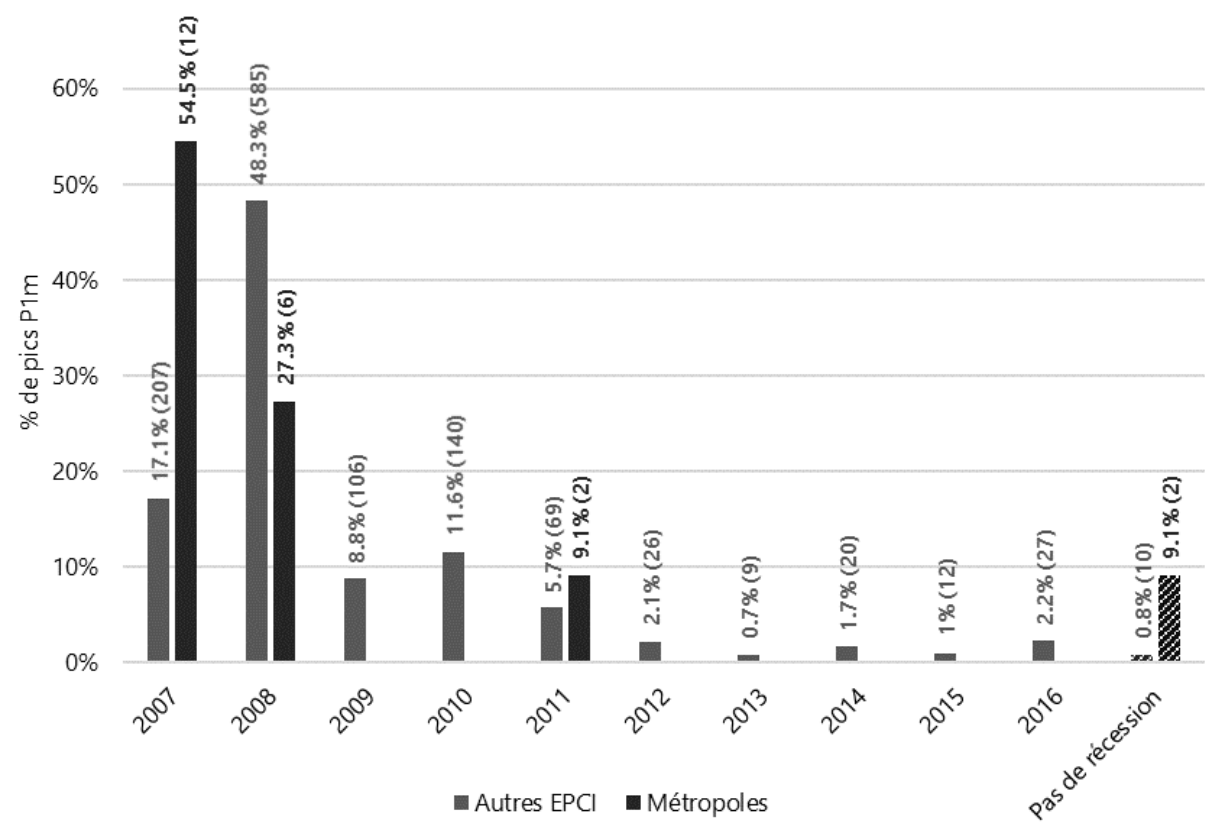

Source : Auteurs, Acoss et REE-Insee. 
Figure 7. Nombre d'EPCI connaissant une première croissance de l'emploi privé par an

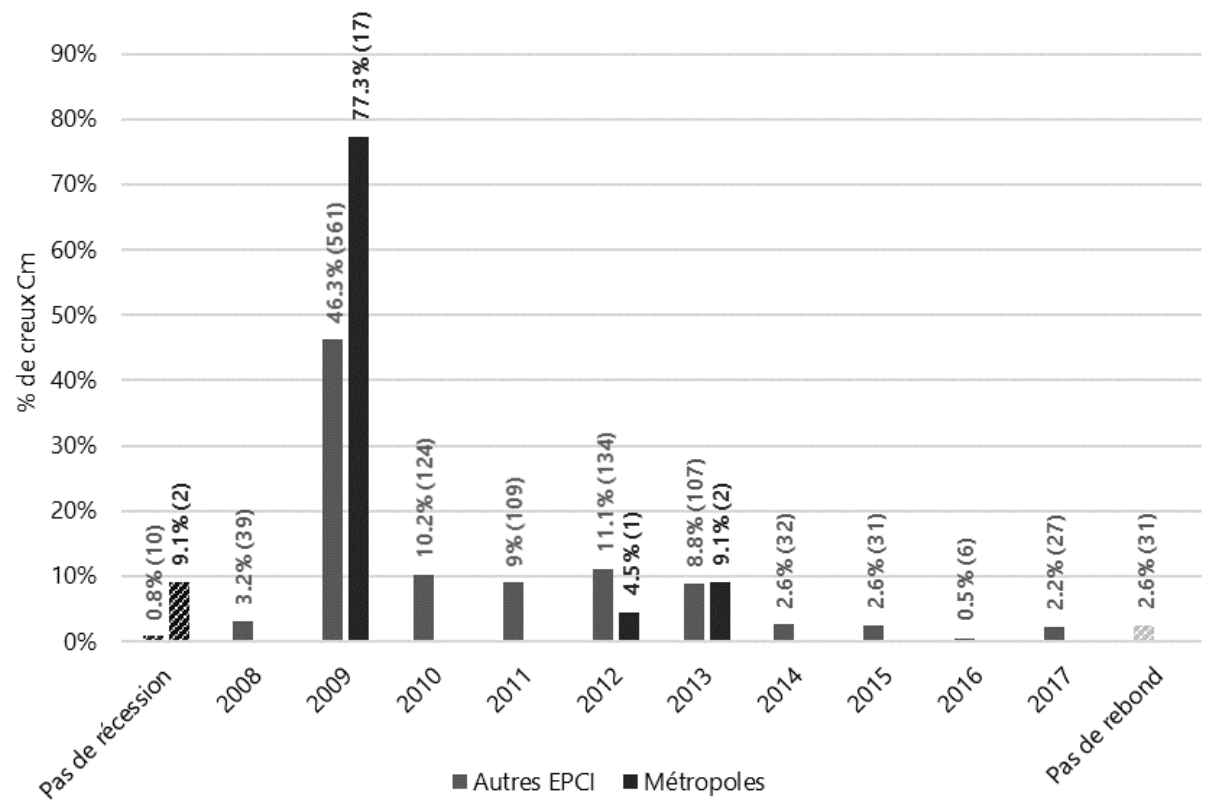

Source : Auteurs, Acoss et REE-Insee.

Figure 8. Nombre d'EPCI ayant récupéré leur niveau d'emploi privé d'avant la crise par an

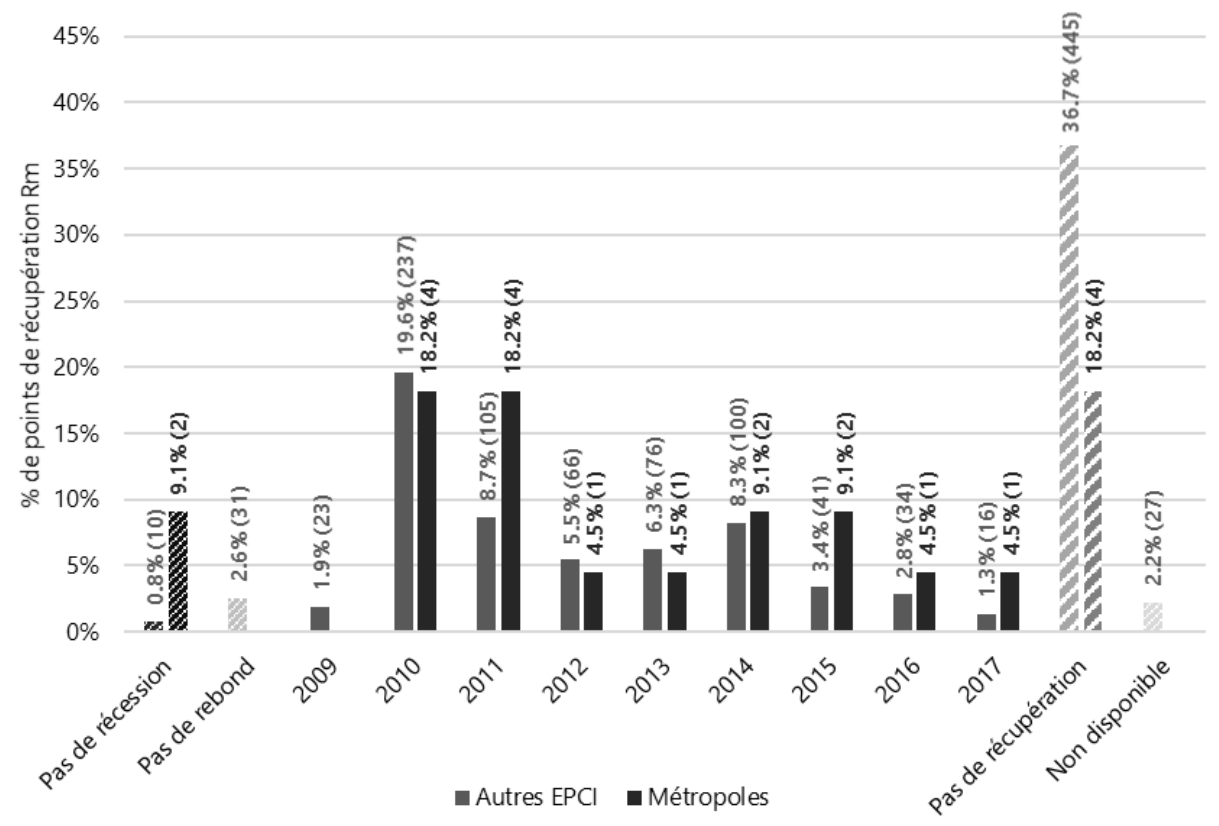

Source : Auteurs, Acoss et REE-Insee.

Ces résultats montrent à quel point l'enjeu de longue période est important lorsque l'on s'intéresse à la résilience. Mesurer les intensités de déclin et de reprise ne suffit pas à déterminer la capacité de récupération des territoires. L'analyse géographique de ces variables apportent des éléments de compréhension. 
Carte 4. Date du début du déclin, de la croissance et de la récupération économique dans les EPCI français

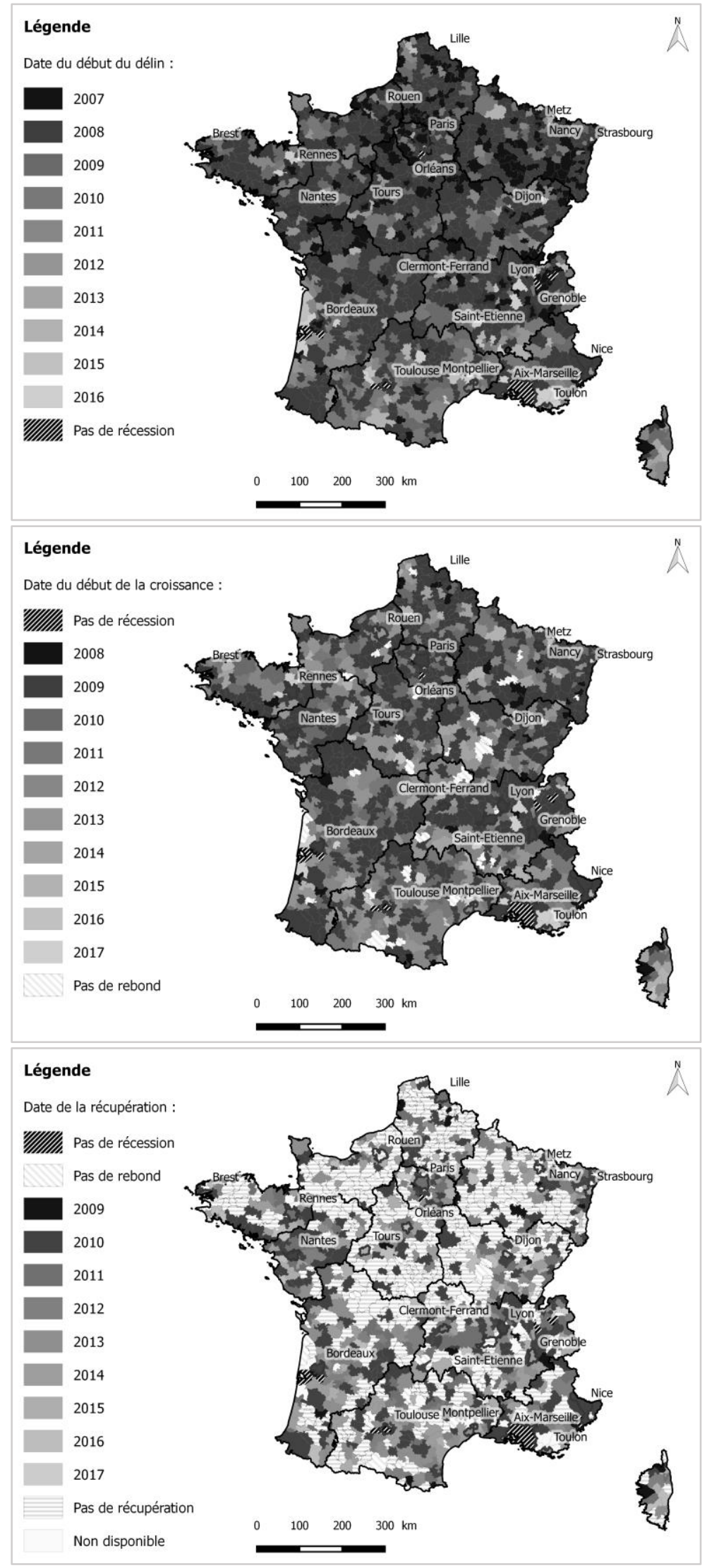

Source : Auteurs, Acoss et REE-Insee. 
Les régions de tradition industrielle du Nord-Est, telles que le Grand Est, les Hauts-de-France, la Bourgogne-Franche-Comté et l'Auvergne-Rhône-Alpes, ont été parmi les premières à connaître une baisse de l'emploi privé (Carte 4). Les métropoles sont également proportionnellement plus nombreuses à être touchées par la crise dès 2007. C'est notamment le cas de Grenoble, Metz, Dijon, Bordeaux, Rennes, Tours, Saint-Etienne, Orléans, Nancy, Strasbourg, Paris et Lyon.

Puis, les effets du choc économique s'accélèrent en 2008, s'intensifiant dans les régions précédemment atteintes et touchant en plus les régions du Sud-Ouest, comme la NouvelleAquitaine, l'Occitanie, le Centre-Val de Loire et les Pays de la Loire. Les 6 métropoles touchées en 2008 sont celles de Rouen, Nice, Brest, Nantes, Lille et Clermont.

Comme on l'a déjà remarqué plus haut, 4 métropoles ne subissent pas la crise économique au cours de la période 2007-2009. Montpellier et Toulon résistent jusqu'en 2011, tandis que Toulouse et Aix-Marseille n'ont connu aucune récession de leurs emplois privés sur toute la période étudiée. Ces premiers éléments soulignent donc la diversité des situations en termes de point de départ de la crise. Qu'en est-il du rebond et de la récupération ?

La géographie de la reprise est plus homogène. On a vu en effet précédemment qu'une large part des EPCI retrouve une croissance des emplois dès 2009. Cela concerne toutes les régions et tous les types d'intercommunalités (métropoles ou autres EPCI) (Carte 4).

Enfin, la géographie des EPCI, qui en 2017 n'avaient toujours pas récupéré de la crise de 2008, est à nouveau très marquée. On observe en effet une concentration très nette dans la moitié Nord de la France (Carte 4), à l'exception de l'Ile-de-France, des deux départements alsaciens, du littoral atlantique et des deux intercommunalités proches de Rennes et Nantes. Dans ces contextes régionaux structurellement défavorables, les métropoles, on l'a vu, ont subi de fortes pertes d'emplois, et ce dès 2007. En revanche, ce que l'on voit apparaitre ici, c'est une différence importante entre la capacité de récupération des métropoles (date du point $\mathrm{R} m$ ) et celle de leur hinterland.

\subsection{Synthèse des résultats}

En se basant sur la littérature européenne et les quelques travaux français sur le sujet, nous pouvions imaginer que les métropoles étaient mieux armées pour faire face à la crise de 2008, que les autres types d'EPCI, creusant ainsi les disparités entre ces grandes agglomérations et leur périphérie.

L'analyse développée dans cet article apporte des conclusions plus nuancées. Ainsi, les résultats dépendent à la fois des contextes régionaux, mais aussi - ce qui est beaucoup moins étudié - des temporalités de résilience.

La Figure 9 nous aide à synthétiser nos propos. On voit ainsi que les écarts entre les métropoles et autres EPCI ne sont pas constants sur l'ensemble de la période (Figure 9-A). Les métropoles sont plus rapidement et plus intensément touchées par la crise économique que les autres types d'espace. Leur économie, pour partie centrée sur des activités tertiaires mondialisées, est en effet très sensible aux chocs conjoncturels. Ainsi, entre 2007 et 2009, les métropoles sont moins résilientes que les autres espaces. Puis, arrive le moment de la reprise, du retour à la croissance, qui apparait relativement homogène sur l'ensemble du pays. Une très large majorité des EPCI retrouve une croissance des emplois en 2009 ou 2010. C'est en revanche sur le long terme, durant toute la période de récupération que les métropoles font mieux que les 
autres EPCI. Ainsi, elles retrouvent leur niveau d'emplois de 2007 bien plus rapidement que les autres types d'espace. Le deuxième résultat important tient aux contextes régionaux. Ainsi, selon les régions, la résilience des intercommunalités et notamment les écarts entre métropoles et périphérie diffèrent. Ces variations ne sont, là encore, pas homogènes tout au long de la période observée. La Figure 9-B mesure les écarts-types pondérés (ou la variabilité d'un EPCI à l'autre) des variations de l'emploi à l'intérieur d'une même région et pour la France entière. Des écarts négatifs indiquent, par exemple, une certaine homogénéité régionale, que ce soit sur l'ensemble des EPCI ou entre les métropoles.

La phase de déclin (2007-2009) est régionalement très marquée, à savoir que les taux de perte d'emplois sont très proches d'un EPCI à l'autre au sein d'une même région, mais très différents d'une région à l'autre. Ce résultat s'observe partout sauf en Corse, en Auvergne-Rhône-Alpes et en Occitanie, moins touchées durant cette période de déclin. On observe entre 2007 et 2009 un creusement des inégalités régionales dû à des facteurs structurels. Ceux-ci pénalisent les espaces du Nord de la France, plus productifs et, très souvent encore industriels, voire manufacturiers. On a ainsi vu que ces régions concentrent les trois quarts des EPCI à faible résilience ! Durant cette période, les métropoles de ces régions sont autant touchées, voire même plus, que les autres intercommunalités. Les régions méridionales, structurellement plus résidentielles et touristiques, sont plus protégées, que ce soit dans les métropoles ou en dehors.

La période 2009-2013 est relativement homogène, avec des reprises économiques observables un peu partout, à la fois régionalement et pour une large part des EPCI, et quasiment toutes les métropoles.

On retrouve, enfin, un effet régional tout au long de la période de récupération, soit après 2013. Plus d'un tiers des EPCI n'avaient pas récupéré leur niveau d'emplois en 2017. Ces EPCI sont à nouveau situés dans une moitié Nord de la France, mais ils sont, cette fois, beaucoup plus nombreux dans les intercommunalités non métropolitaines. Ainsi, dans les espaces non métropolitains, plus ruraux, des régions du Nord de la France, on observe un double creusement des inégalités, causé par des effets structurels régionaux dès le début de la crise et des écarts croissants entre les zones denses urbaines et celles plus périphériques et rurales en période de récupération. Pour autant, ces territoires ne sont pas condamnés au déclin et la reprise économique a bien eu lieu. Mais, les pertes d'emplois ont été telles que la récupération n'a, pour l'heure, été que partielle. Ces espaces ont retrouvé un chemin de croissance, mais non suffisant pour parvenir à combler la crise économique de 2008. 
Figure 9. Comparaison des intensités et temporalités de résilience entre EPCI, Métropoles et régions

Figure 9-A. Écart entre les moyennes des variations d'emplois privés des métropoles et des autres EPCI.

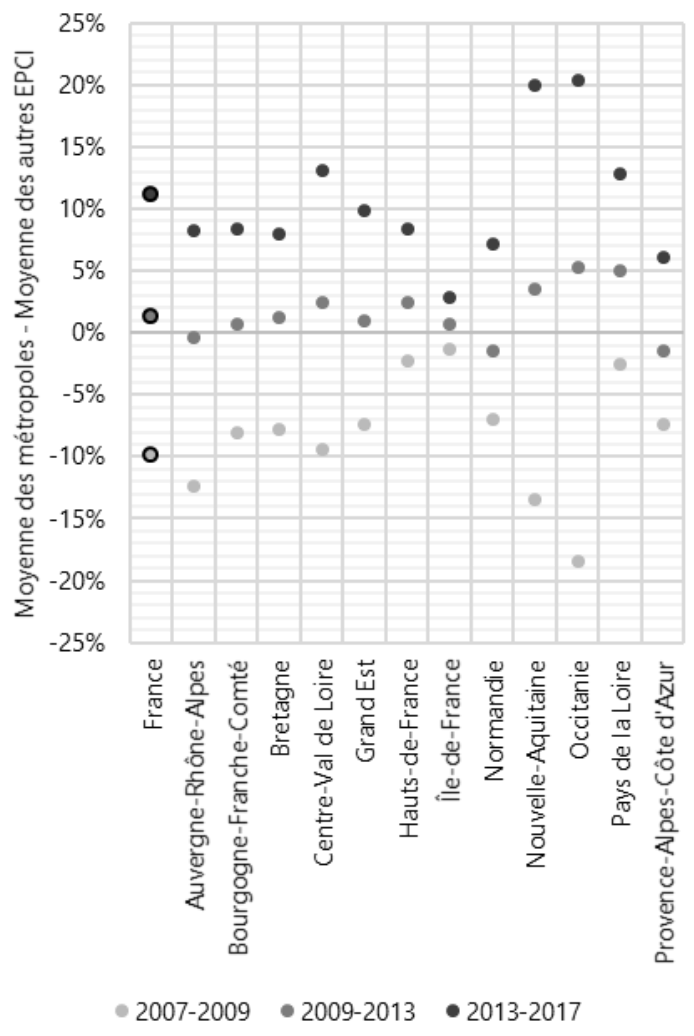

Figure 9-B. Écart entre les écart-types des variations d'emplois privés des EPCI et des métropoles au sein des régions et dans l'ensemble de la France.

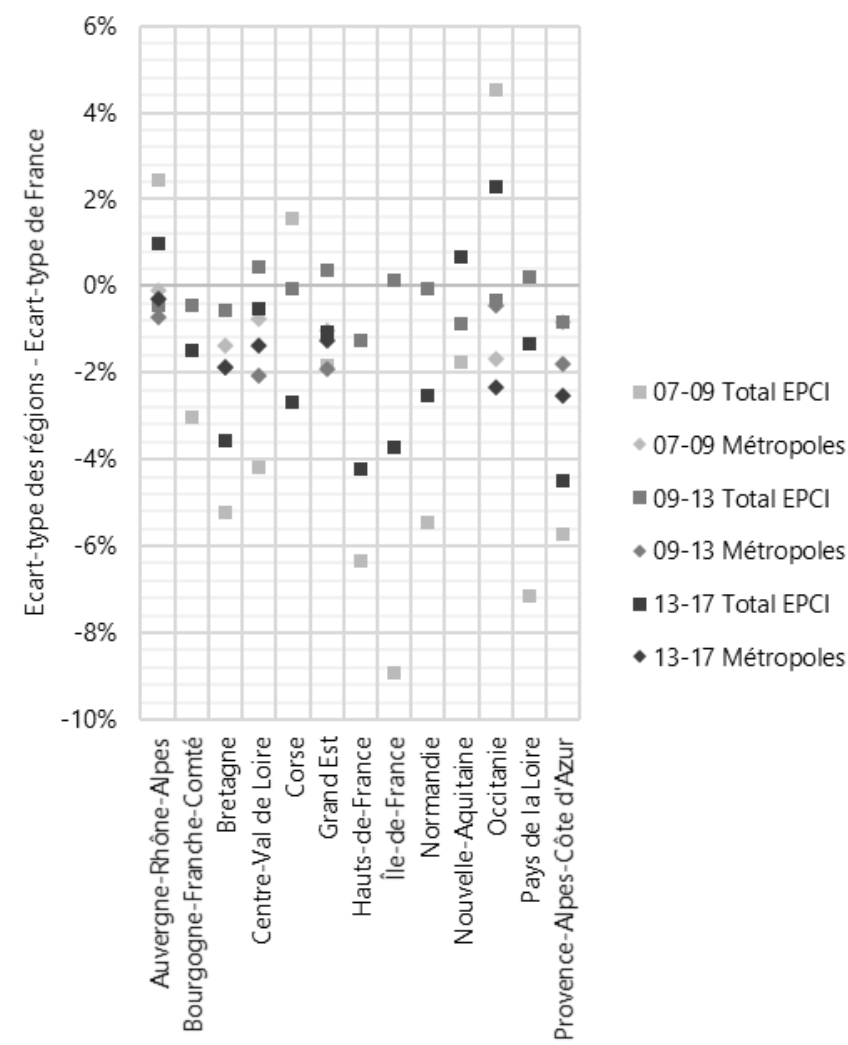

Source : Auteurs, Acoss et REE-Insee.

Note : La variation des emplois privés de chaque EPCI a été calculée entre 2007 et 2009, entre 2009 et 2013 et entre 2013 et 2017. • La Figure 9-A représente la différence entre la moyenne des métropoles et la moyenne des autres EPCI, en distinguant les trois phases. Une valeur positive indique qu'en moyenne les métropoles connaissent des variations d'emplois supérieures à celles des autres EPCI. • La Figure 9-B représente la différence entre l'écart-type de chacune des régions et l'écart-type de la France, en distinguant les trois phases ainsi que les métropoles de l'ensemble des EPCI. Une valeur négative indique qu'il y a moins d'écart entre les variations d'emplois des EPCI au sein d'une même région que dans l'ensemble de la France. 


\section{$-5-$ \\ Conclusion}

En conclusion, quelles leçons pouvons-nous tirer de la crise de 2008 pour la période actuelle ? La crise socio-économique liée à la COVID-19 est évidemment très différente, à la fois dans ses causes et dans sa composante structurelle. On sait cependant qu'elle impacte déjà les pays européens de façon différenciée. Qu'en sera-t-il aux échelles infranationales ? Les premiers chiffres nationaux montrent que certains secteurs sont plus touchés que d'autres (commerces, tourisme, services marchands, par exemple). Ces secteurs, contrairement à la crise de 2008, sont présents dans la plupart des territoires, tout au moins en France. La régionalisation du choc observée en 2008 pourrait donc être moins marquée et ne pas pénaliser exclusivement les régions productives et industrielles (qui, rappelons-le, n'avaient pas encore récupéré de la crise précédente). Par contre, ce que l'on appelle souvent l'économie résidentielle et touristique, qui a joué un rôle d'amortisseur majeur en 2008, est cette fois beaucoup plus vulnérable. Les fortes pertes d'emplois constatées à ce jour dans ce type de secteurs sont inquiétantes, socialement et spatialement. On sait que ces activités embauchent massivement des personnes peu ou pas qualifiées, beaucoup plus de femmes que d'hommes, et constituent très souvent la dernière source d'emplois locaux et revenus d'activités quand les autres secteurs se sont retirés. La fragilité - nouvelle - de cette économie renforce l'importance de privilégier un développement équilibré, ou productivo-résidentiel, des territoires.

Les résultats obtenus montrent aussi que les métropoles sont extrêmement exposées aux chocs conjoncturels et subissent très souvent la crise, à la fois plus tôt et plus intensément que les autres territoires. Ainsi, à l'image de ce que l'on observe avec la Covid-19, les espaces denses et urbains perdront (en proportion) sans doute plus d'emplois au moment du choc que les autres territoires. Pour autant, l'exemple de 2008 nous rappelle qu'il est trop tôt pour en tirer des conclusions sur un éventuel déclin des villes ou des métropoles. En effet, nous avons vu que la résilience est un processus de long terme et les périodes de reprise, puis de récupération sont fondamentales. En la matière, les métropoles, contextuellement désavantagées au départ, sont capables de compenser dans la durée les pertes grâce à leurs facteurs compositionnels (économie diversifiée, secteurs moins impactés, revenu des ménages plus élevé) et collectifs (réseaux, connexion, infrastructures). Enfin, des territoires spécialisés dans des activités épargnées par la crise peuvent très bien s'en sortir aussi. En revanche, cette spécialisation est plus risquée à plus longue échéance, à l'image des hauts lieux du tourisme, totalement épargnés en 2008, mais durement frappés en 2020, ou bien encore de petites villes spécialisées dans une industrie particulière (l'aéronautique, par exemple).

Enfin, dans les régions les plus touchées en 2008, on observe un décrochage, un décalage, entre la capacité de récupération des métropoles et celle des espaces plus ruraux ou périphériques. Dans la plupart de ces espaces, l'emploi est certes reparti à la hausse après 2009, mais plus doucement qu'ailleurs, ne suffisant pas à combler les pertes. Ici, la crise de la COVID19 ne sera peut-être pas plus forte qu'ailleurs, mais elle frappera des espaces (et des ménages) déjà fortement précarisés au démarrage de la pandémie. Espérons que ces territoires, extrêmement vulnérables, attirent l'attention des décideurs et trouvent de nouvelles trajectoires de changement et de résilience. 


\section{Bibliographie}

Barca F (2009) Agenda for a reformed cohesion policy. Brussels: European Communities.

Baude J (2016) Les métropoles créent-elles plus d'emplois ?. EchoGéo, (38).

Borzic M, Le Jeannic T (2014) En matière d'emploi, les métropoles ont davantage résisté à la crise.

Bouba-Olga O, Grossetti, M (2015) La métropolisation, horizon indépassable de la croissance économique ? Revue de l'OFCE, 143(7): 117- 144.

Brakman S, Garretsen H, van Marrewijk C (2015) Regional resilience across Europe: On urbanisation and the initial impact of the Great Recession. Cambridge Journal of Regions, Economy and Society, 8(2): 225- 240.

Bristow G, Healy A (2018) Economic crisis and the resilience of regions: A European study. Edward Elgar Publishing.

Bouba-Olga O, Chauchefoin P, Chiron H, Ferru M (2017) Dynamiques territoriales : éloge de la diversité. Atlantique, éditions de L'Actualité scientifique Nouvelle-Aquitaine.

Brown, L, Greenbaum R T (2016) The role of industrial diversity in economic resilience: An empirical examination across 35 years. Urban Studies, 54(6): 1347- 1366.

Camagni R, Capello R (2015) Second-rank city dynamics: Theoretical interpretations behind their growth potentials.

Capello R, Caragliu A (2016) After crisis scenarios for Europe: alternative evolutions of structural adjustments. Cambridge Journal of Regions, Economy and Society, 9(1): 81-101.

Capello R, Caragliu A, Fratesi U (2015) Spatial heterogeneity in the costs of the economic crisis in Europe: are cities sources of regional resilience?. Journal of Economic Geography, 15(5): 951-972.

Christopherson S, Clark G L, Whiteman J (2015) Introduction: The Euro crisis and the future of Europe. Journal of Economic Geography, 15(5): 843-853.

Coffano M, Foray D (2014) The centrality of entrepreneurial discovery in building and implementing a smart specialisation strategy. Scienze Regionali.

Crescenzi R, Rodríguez-Pose A (2011) Innovation and regional growth in the European Union. Springer-Verlag.

Crescenzi R, Luca D, Milio S (2016) The geography of the economic crisis in Europe: national macroeconomic conditions, regional structural factors and short-term economic performance. Cambridge Journal of Regions, Economy and Society, 9(1): 13-32.

Danson M (2002) Small firm formation and regional economic development. Routledge.

Davezies L (2012) La crise qui vient. La nouvelle fracture territoriale. Seuil.

Di Caro P (2015) Testing and explaining economic resilience with an application to Italian regions. Papers in Regional Science, 96(1): 93- 113.

Dijkstra L, Garcilazo E, McCann P (2015) The effects of the global financial crisis on European regions and cities. Journal of Economic Geography, 15(5): 935- 949. 
Diodato D, Weterings A B (2015) The resilience of regional labour markets to economic shocks: Exploring the role of interactions among firms and workers. Journal of Economic Geography, 15(4): 723-742.

Dissart J C (2003) Regional economic diversity and regional economic stability: Research results and agenda. International Regional Science Review, 26(4): 423-446.

Donald B, Glasmeier A, Gray M, Lobao L (2014) Austerity in the city: economic crisis and urban service decline? Cambridge Journal of Regions, Economy and Society, 7(1): 3- 15.

Eraydin A (2016. Attributes and characteristics of regional resilience: Defining and measuring the resilience of Turkish regions. Regional Studies, 50(4): 600- 614.

Faggian A, McCann P (2009) Human capital and regional development. In: Capello R, Nijkamp P (eds) Handbook of Regional Growth and Development Theories. Edward Elgar: 131-151.

FNAU (2011) L'emploi local face à la crise 2008 à 2009 : Un révélateur des divergence territoriales.

Fratesi U, Perucca G (2018) Territorial capital and the resilience of European regions. The Annals of Regional Science, 60(2): 241-264.

Fratesi U, Rodríguez-Pose A (2016) The crisis and regional employment in Europe: What role for sheltered economies? Cambridge Journal of Regions, Economy and Society, 9(1): 33- 57.

Giannakis E, Bruggeman A (2015) Economic crisis and regional resilience: Evidence from Greece. Papers in Regional Science, 96(3): 451- 476.

Giannakis E, Bruggeman A (2017) Determinants of regional resilience to economic crisis: A European perspective. European Planning Studies, 25(8): 1394- 1415.

Glaeser E L, Ponzetto G A M, Tobio K (2014) Cities, skills and regional change. Regional Studies, 48(1): 7- 43.

Groot S P, Möhlmann J L, Garretsen J H, de Groot H L (2011) The crisis sensitivity of European countries and regions: stylized facts and spatial heterogeneity. Cambridge Journal of Regions, Economy and Society, 4(3): 437-456.

Hamdouch A (2012) Mondialisation et résilience des territoires : Trajectoires, dynamiques d'acteurs et expériences. PUQ.

Hassink R (2010) Regional resilience: a promising concept to explain differences in regional economic adaptability?. Cambridge journal of regions, economy and society, 3(1): 45-58.

Lane P R, Milesi-Ferretti G M (2010) The cross-country incidence of the global crisis. IMF Economic Review, 59(1): 77- 110.

Manca A R, Benczur P, Giovannini E (2017) Between policy and practice: A conceptual framework for resilience in EU policy making. JRC Working Papers in Economics and Finance, Forthcoming.

Martin R (2010) The local geographies of the financial crisis: From the housing bubble to economic recession and beyond. Journal of Economic Geography, 11(4): 587- 618.

Martin R (2012) Regional economic resilience, hysteresis and recessionary shocks. Journal of Economic Geography, 12(1): 1- 32.

Martin R, Sunley P (2015) On the notion of regional economic resilience: Conceptualization and explanation. Journal of Economic Geography, 15(1): 1- 42. 
Martin R, Sunley P, Gardiner B, Tyler P (2016) How regions react to recessions: Resilience and the role of economic structure. Regional Studies, 50(4): 561- 585.

McCann P, Ortega-Argilés O (2014) The role of the smart specialisation agenda in a reformed EU cohesion policy. Scienze Regionali.

Östh J, Reggiani A, Galiazzo G (2015) Spatial economic resilience and accessibility: A joint perspective. Computers, Environment and Urban Systems, 49: 148- 159.

Pendall R, Foster K A, Cowell M (2007) Resilience and regions: Building understanding of the metaphor. Cambridge Journal of Regions, Economy and Society, 3(1): 71-84.

Pontarollo N, Serpieri C (2020) A composite policy tool to measure territorial resilience capacity. Socio-Economic Planning Sciences, 70: 100669.

Psycharis Y, Kallioras D, Pantazis P (2014) Economic crisis and regional resilience: Detecting the 'geographical footprint' of economic crisis in Greece. Regional Science Policy \& Practice, 6(2): 121- 141.

Reinhart C M, Rogoff K S (2009) The aftermath of financial crises. American Economic Review, 99(2): 466-72.

Sapir A, Aghion P, Bertola G, Hellwig M, Pisani-Ferry J, Rosati D, ... Smith P M (2004) An agenda for a growing Europe: The Sapir report. OUP Oxford.

Storper M, Scott A J (2009) Rethinking human capital, creativity and urban growth. Journal of Economic Geography, 9(2): 147- 167. 


\section{Annexes}

Tableau 2. Cycle de résilience de quelques métropoles

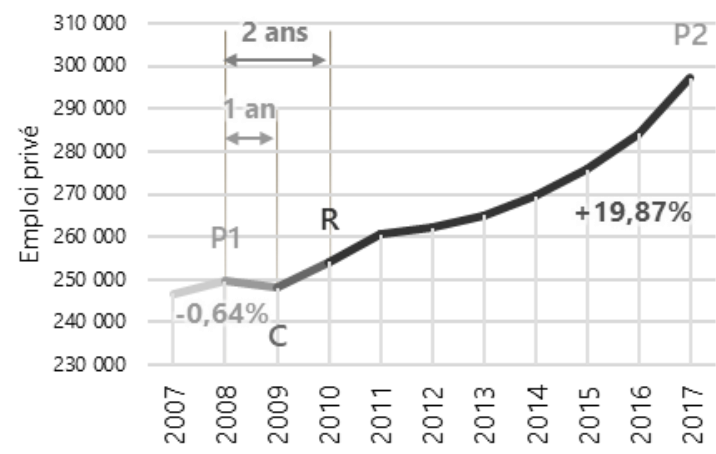

Nantes Métropole.

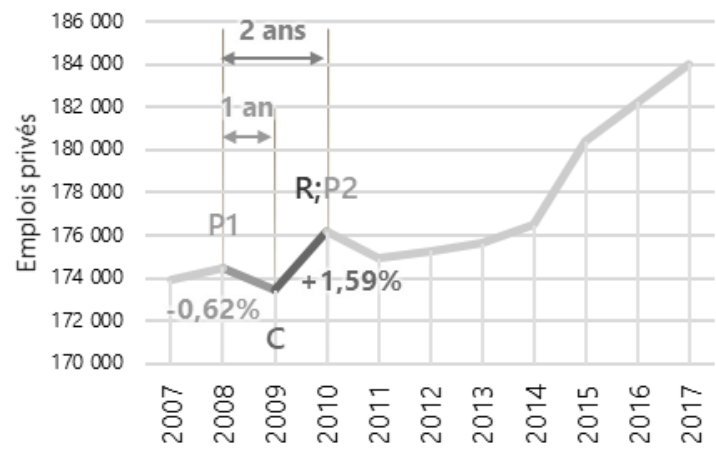

Métropole Nice Côte d'Azur.

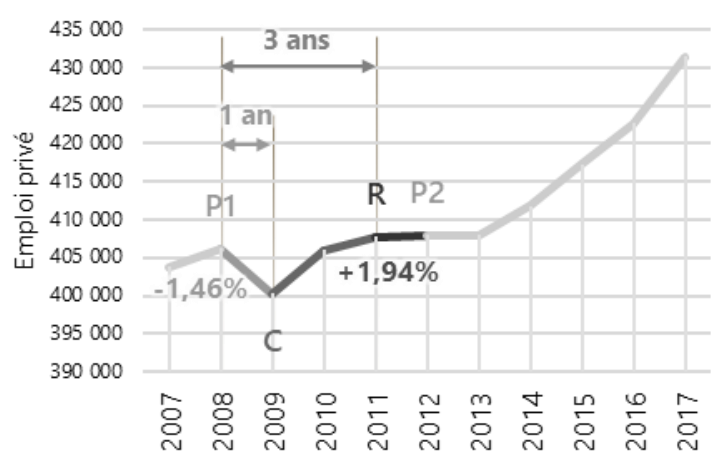

Métropole Européenne de Lille.

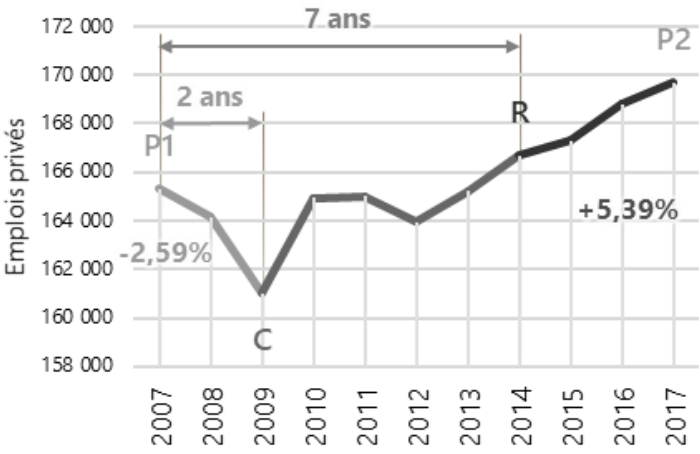

Grenoble-Alpes-Métropole.

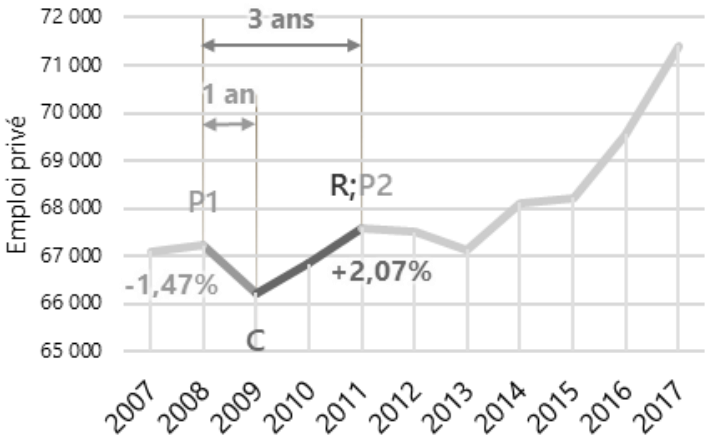

Brest Métropole

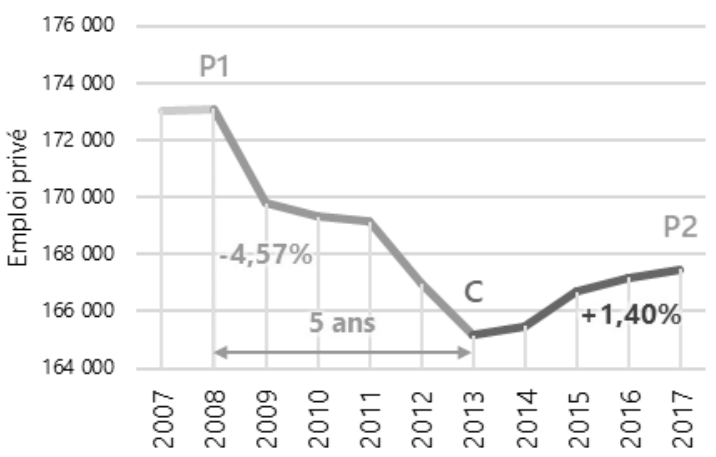

Métropole Rouen Normandie.

Source : Auteurs, Acoss et REE-Insee. 
Tableau 3. Intensité du déclin et de la croissance d'emplois privés par type d'EPCI et par tranche de population totale (2016)

\begin{tabular}{|c|c|c|c|c|c|c|c|}
\hline & $\begin{array}{l}\text { Nombre } \\
\text { d'EPCI }\end{array}$ & $\begin{array}{l}\text { Déclin } \\
\text { moyen } \\
(\text { en \%) }\end{array}$ & $\begin{array}{c}\text { Médiane } \\
\text { du déclin } \\
\text { (en \%) }\end{array}$ & $\begin{array}{l}\text { Écart-type } \\
\text { du déclin } \\
\text { (en \%) }\end{array}$ & $\begin{array}{c}\text { Croissance } \\
\text { moyenne } \\
(\text { en \%) }\end{array}$ & $\begin{array}{l}\text { Médiane } \\
\text { de la } \\
\text { croissance } \\
\text { (en \%) }\end{array}$ & $\begin{array}{l}\text { Écart-type } \\
\text { de la } \\
\text { croissance } \\
\text { (en \%) }\end{array}$ \\
\hline Ensemble des EPCI & 1236 & $-3,99 \%$ & $-2,89 \%$ & $3,90 \%$ & $4,58 \%$ & $2,90 \%$ & $5,07 \%$ \\
\hline Communautés de Com. & 993 & $-4,33 \%$ & $-1,28 \%$ & $4,21 \%$ & $4,75 \%$ & $1,35 \%$ & $5,24 \%$ \\
\hline$<10000$ hab. & 210 & $-4,79 \%$ & $-0,79 \%$ & $4,85 \%$ & $4,36 \%$ & $1,00 \%$ & $4,26 \%$ \\
\hline 10 à 25000 hab. & 443 & $-4,63 \%$ & $-1,50 \%$ & $4,36 \%$ & $4,18 \%$ & $1,31 \%$ & $4,42 \%$ \\
\hline 25 à 55000 hab. & 315 & $-3,74 \%$ & $-1,29 \%$ & $3,57 \%$ & $5,66 \%$ & $1,64 \%$ & $6,44 \%$ \\
\hline 55 à 80000 hab. & 21 & $-3,25 \%$ & $-0,79 \%$ & $2,92 \%$ & $6,68 \%$ & $1,51 \%$ & $7,20 \%$ \\
\hline 80 à 150000 hab. & 4 & $-1,45 \%$ & $-1,09 \%$ & $0,90 \%$ & $5,50 \%$ & $4,01 \%$ & $5,45 \%$ \\
\hline Communautés d'Agglo. & 208 & $-2,97 \%$ & $-1,93 \%$ & $2,54 \%$ & $3,88 \%$ & $2,04 \%$ & $4,35 \%$ \\
\hline 25 à 55000 hab. & 29 & $-3,38 \%$ & $-2,76 \%$ & $2,42 \%$ & $3,13 \%$ & $1,79 \%$ & $3,72 \%$ \\
\hline 55 à 80000 hab. & 69 & $-3,40 \%$ & $-1,71 \%$ & $2,93 \%$ & $3,52 \%$ & $1,74 \%$ & $3,53 \%$ \\
\hline 80 à 150000 hab. & 76 & $-2,82 \%$ & $-1,93 \%$ & $2,47 \%$ & $4,20 \%$ & $2,07 \%$ & $4,83 \%$ \\
\hline 150 à 450000 hab. & 34 & $-2,15 \%$ & $-1,25 \%$ & $1,81 \%$ & $4,53 \%$ & $2,40 \%$ & $5,11 \%$ \\
\hline Communautés Urbaines & 13 & $-3,37 \%$ & $-3,10 \%$ & $1,51 \%$ & $2,62 \%$ & $1,37 \%$ & $1,82 \%$ \\
\hline 55 à 80000 hab. & 1 & & & & & & \\
\hline 80 à 150000 hab. & 2 & $-3,71 \%$ & $-3,71 \%$ & $0,36 \%$ & $2,77 \%$ & $2,77 \%$ & $2,75 \%$ \\
\hline 150 à 450000 hab. & 10 & $-3,31 \%$ & $-2,89 \%$ & $1,66 \%$ & $2,59 \%$ & $1,79 \%$ & $1,79 \%$ \\
\hline Métropoles de droit com. & 19 & $-2,48 \%$ & $-2,10 \%$ & $1,57 \%$ & $5,70 \%$ & $2,65 \%$ & $5,81 \%$ \\
\hline 150 à 450000 hab. $^{(1)}$ & 9 & $-3,09 \%$ & $-2,56 \%$ & $1,60 \%$ & $4,01 \%$ & $2,91 \%$ & $2,76 \%$ \\
\hline 450 à 700000 hab. ${ }^{(2)}$ & 7 & $-2,17 \%$ & $-2,15 \%$ & $1,47 \%$ & $6,44 \%$ & $2,65 \%$ & $7,02 \%$ \\
\hline 700k à 1000000 hab. ${ }^{(3)}$ & 2 & $-0,56 \%$ & $-0,28 \%$ & & $18,57 \%$ & $9,28 \%$ & \\
\hline$>1000000$ hab. ${ }^{(4)}$ & 1 & $-1,46 \%$ & $-1,46 \%$ & & $1,94 \%$ & $1,94 \%$ & \\
\hline Métropoles à statut part. & 2 & $-1,32 \%$ & $-0,66 \%$ & & $10,98 \%$ & $5,49 \%$ & \\
\hline$>1000000$ hab. $^{(5)}$ & 2 & $-1,32 \%$ & $-0,66 \%$ & & $10,98 \%$ & $5,49 \%$ & \\
\hline Métropole de Lyon & 1 & $-1,14 \%$ & $-1,14 \%$ & & $15,34 \%$ & $15,34 \%$ & \\
\hline > 1000000 hab. & 1 & $-1,14 \%$ & $-1,14 \%$ & & $15,34 \%$ & $15,34 \%$ & \\
\hline
\end{tabular}

(1) Metz Métropole, Dijon Métropole, Brest Métropole, Tours Métropole Val de Loire, Saint-Etienne Métropole, Orlean: Métropole, Métropole du Grand Nancy, Clermont Auvergne Métropole, Métropole Toulon-Provence-Méditerranée

(2) Métropole Rouen Normandie, Métropole Nice Cote d'Azur, Grenoble-Alpes-Métropole, Montpellier Méditerrané Métropole, Rennes Métropole, Nantes Métropole, Eurométropole de Strasbourg

(3) Toulouse Métropole, Bordeaux Métropole

(4) Métropole Européenne de Lille

(5) Métropole du Grand Paris, Métropole d'Aix-Marseille-Provence

Sources : IGN 2019 ; Insee, Recensement de la population 2016 ; Acoss \& REE-Insee 2016, calcul des auteurs. 
Tableau 4. Intensité du déclin et de la croissance d'emplois privés des métropoles dans leurs régions

\begin{tabular}{|c|c|c|c|}
\hline & $\begin{array}{l}\text { Nombre } \\
\text { d'EPCI }\end{array}$ & $\begin{array}{l}\text { Déclin moyen } \\
\quad(\text { en \%) }\end{array}$ & $\begin{array}{l}\text { Croissance } \\
\text { moyenne } \\
\text { (en \%) }\end{array}$ \\
\hline Auvergne-Rhône-Alpes & 170 & $-1,42 \%$ & $7,28 \%$ \\
\hline \multicolumn{2}{|l|}{ Métropole de Lyon } & $-1,14 \%$ & $15,34 \%$ \\
\hline \multicolumn{2}{|l|}{ Clermont Auvergne Métropole } & $-1,37 \%$ & $7,94 \%$ \\
\hline \multicolumn{2}{|l|}{ Grenoble-Alpes-Métropole } & $-2,59 \%$ & $5,39 \%$ \\
\hline \multicolumn{2}{|l|}{ Saint-Etienne Métropole } & $-5,79 \%$ & $1,65 \%$ \\
\hline Bourgogne-Franche-Comté & 113 & $-1,75 \%$ & $1,05 \%$ \\
\hline \multicolumn{2}{|l|}{ Dijon Métropole } & $-2,58 \%$ & $3,18 \%$ \\
\hline Bretagne & 59 & $-1,13 \%$ & $1,74 \%$ \\
\hline \multicolumn{2}{|l|}{ Brest Métropole } & $-1,47 \%$ & $2,07 \%$ \\
\hline \multicolumn{2}{|l|}{ Rennes Métropole } & $-2,44 \%$ & $2,65 \%$ \\
\hline Centre-Val de Loire & 81 & $-1,76 \%$ & $0,86 \%$ \\
\hline \multicolumn{2}{|l|}{ Tours Métropole Val de Loire } & $-2,10 \%$ & $5,49 \%$ \\
\hline \multicolumn{2}{|l|}{ Orléans Métropole } & $-4,53 \%$ & $7,90 \%$ \\
\hline Corse & 19 & Récession ap. crise & (1) \\
\hline Grand Est & 149 & $-1,91 \%$ & $1,27 \%$ \\
\hline \multicolumn{2}{|l|}{ Eurométropole de Strasbourg } & $-2,15 \%$ & $7,71 \%$ \\
\hline \multicolumn{2}{|l|}{ Métropole du Grand Nancy } & $-2,56 \%$ & $0,95 \%$ \\
\hline \multicolumn{2}{|l|}{ Metz Métropole } & $-4,28 \%$ & $2,91 \%$ \\
\hline Hauts-de-France & 91 & $-1,91 \%$ & $1,53 \%$ \\
\hline \multicolumn{2}{|l|}{ Métropole Européenne de Lille } & $-1,46 \%$ & $1,94 \%$ \\
\hline Île-de-France & 52 & $-1,22 \%$ & $10,08 \%$ \\
\hline \multicolumn{2}{|l|}{ Métropole du Grand Paris } & $-1,32 \%$ & $10,98 \%$ \\
\hline Normandie & 72 & $-1,59 \%$ & $0,53 \%$ \\
\hline \multicolumn{2}{|l|}{ Métropole Rouen Normandie } & $-4,57 \%$ & $1,40 \%$ \\
\hline Nouvelle-Aquitaine & 153 & $-0,78 \%$ & $2,15 \%$ \\
\hline \multicolumn{2}{|l|}{ Bordeaux Métropole } & $-0,56 \%$ & $18,57 \%$ \\
\hline Occitanie & 161 & Récession ap. crise & $(2)$ \\
\hline \multicolumn{2}{|l|}{ Toulouse Métropole } & Aucune récession & $21,64 \%$ \\
\hline \multicolumn{2}{|l|}{ Montpellier Méditerranée Métropole } & Récession ap. crise & (3) $13,09 \%$ \\
\hline Pays de la Loire & 68 & $-1,53 \%$ & $8,19 \%$ \\
\hline \multicolumn{2}{|l|}{ Nantes Métropole } & $-0,64 \%$ & $19,87 \%$ \\
\hline Provence-Alpes-Côte d'Azur & 48 & Aucune récession & $10,50 \%$ \\
\hline \multicolumn{2}{|l|}{ Métropole d'Aix-Marseille-Provence } & Aucune récession & $11,87 \%$ \\
\hline \multicolumn{2}{|l|}{ Métropole Toulon-Provence-Méditerranée } & Récession ap. crise & (4) $5,73 \%$ \\
\hline \multicolumn{2}{|l|}{ Métropole Nice Côte d'Azur } & $-0,62 \%$ & $1,59 \%$ \\
\hline
\end{tabular}

Cas particuliers de récession après la crise :

(1) Corse : $\quad$ +22,21\% de 2007 à 2016, -2,01\% de 2016 à 2017

(2) Occitanie : $\quad+13,83 \%$ de 2007 à 2016, -2,28\% de 2016 à 2017

(3) Montpellier : +4,77\% de 2007 à 2011, -0,50\% de 2011 à 2012, +13,09\% de 2012 à 2017

(4) Toulon : +4,79\% de 2007 à 2011, - $\quad$ - +,47\% de 2011 à 2013, +5,73\% de 2013 à 2017

Sources : IGN 2019 ; Insee, Recensement de la population 2016 ; Acoss \& REE-Insee 2016, calcul des auteurs. 